\title{
Representation of model error in a convective-scale ensemble prediction system
}

\author{
L. H. Baker, A. C. Rudd, S. Migliorini, and R. N. Bannister \\ Department of Meteorology, University of Reading, Earley Gate, P.O. Box 243, Reading, RG6 6BB, UK
}

Correspondence to: L. H. Baker (1.h.baker@ reading.ac.uk)

Received: 27 June 2013 - Revised: 29 November 2013 - Accepted: 10 December 2013 - Published: 8 January 2014

\begin{abstract}
In this paper ensembles of forecasts (of up to six hours) are studied from a convection-permitting model with a representation of model error due to unresolved processes. The ensemble prediction system (EPS) used is an experimental convection-permitting version of the UK Met Office's 24member Global and Regional Ensemble Prediction System (MOGREPS). The method of representing model error variability, which perturbs parameters within the model's parameterisation schemes, has been modified and we investigate the impact of applying this scheme in different ways. These are: a control ensemble where all ensemble members have the same parameter values; an ensemble where the parameters are different between members, but fixed in time; and ensembles where the parameters are updated randomly every 30 or $60 \mathrm{~min}$. The choice of parameters and their ranges of variability have been determined from expert opinion and parameter sensitivity tests. A case of frontal rain over the southern UK has been chosen, which has a multi-banded rainfall structure.

The consequences of including model error variability in the case studied are mixed and are summarised as follows. The multiple banding, evident in the radar, is not captured for any single member. However, the single band is positioned in some members where a secondary band is present in the radar. This is found for all ensembles studied. Adding model error variability with fixed parameters in time does increase the ensemble spread for near-surface variables like wind and temperature, but can actually decrease the spread of the rainfall. Perturbing the parameters periodically throughout the forecast does not further increase the spread and exhibits "jumpiness" in the spread at times when the parameters are perturbed. Adding model error variability gives an improvement in forecast skill after the first $2-3 \mathrm{~h}$ of the forecast for near-surface temperature and relative humidity. For
\end{abstract}

precipitation skill scores, adding model error variability has the effect of improving the skill in the first $1-2 \mathrm{~h}$ of the forecast, but then of reducing the skill after that. Complementary experiments were performed where the only difference between members was the set of parameter values (i.e. no initial condition variability). The resulting spread was found to be significantly less than the spread from initial condition variability alone.

\section{Introduction}

Errors in forecasts originate from a number of sources, namely the initial conditions, the boundary conditions and the model formulation. In synoptic scale forecasts of lead times up to a day, it is thought that the first two sources dominate. However, at convective scale model errors are thought to become more important, especially for relatively short range forecasts. Here we investigate a proposed representation of model error that can influence the forecast skill at convective scale. We use an experimental convection-permitting version of the UK Met Office's 24-member Global and Regional Ensemble Prediction System (EPS) (MOGREPS) (Bowler et al., 2008): the southern UK $1.5 \mathrm{~km}$ EPS (Migliorini et al., 2011; Caron, 2013). We focus on the effect of model error resulting from the parameterisation of unresolved processes; specifically microphysics and turbulent boundary layer processes. In this study we modify the socalled Random Parameters (RP) scheme, used in MOGREPS (Bowler et al., 2008), by applying changes designed to make it appropriate for use in a convective-scale ensemble.

The aim of this work is to investigate how this modified version of the RP scheme affects the characteristics of the ensemble, with particular focus on the ensemble spread and 
the forecast skill. By evaluating these effects, we determine how useful this scheme could be as a method of representing model error in a convective-scale EPS. Although it is difficult to draw general conclusions from one case study, this work presents new results of the impact of a model error scheme on a convection-permitting model.

There are several different methods commonly used to represent model error. In the multiphysics method (also sometimes called the multimodel method), a set of different model physics parameterisation schemes is used, and generally an ensemble is constructed of members which use different combinations of schemes (Stensrud et al., 2000; Berner et al., 2011; Clark Jr. et al., 2010; Clark et al., 2011). The stochastic kinetic energy backscatter (SKEB) scheme of Shutts (2005) (developed further by Berner et al., 2009 and used by Berner et al., 2011) aims to replace upscale kinetic energy lost in the numerical integration of the model and in the parameterisation of unresolved processes. Another method is to use a stochastically perturbed physical tendencies (SPT or SPPT) scheme (Buizza et al., 1999; Bouttier et al., 2012; Fresnay et al., 2012), in which the total tendencies from parameterisation schemes are perturbed. A further method, and the one on which we focus here, is to perturb a set of individual parameters within the parameterisation schemes themselves. This method can be applied in two different ways: the first is to perturb individual or sets of parameters about their default values, and keep these perturbed values fixed throughout each forecast; the second is to use a stochastic technique to vary the parameter values periodically throughout the forecast. The fixed parameter perturbation method has been widely used for global ensembles (notably the climateprediction.net multi-thousand member ensemble project (Murphy et al., 2004; Stainforth et al., 2005) and the Met Office's Quantifying Uncertainty in Model Predictions (QUMP) ensemble (Murphy et al., 2004)), mesoscale ensemble systems (Hacker et al., 2011) and convection-permitting models (Gebhardt et al., 2011; Vié et al., 2012). In this study we use both methods of setting parameter values (see Sect. 2.2 for details of the scheme used when updating parameters periodically). A similar method was also used by Lin and Neelin (2000) and Bright and Mullen (2002), but for only one and two parameters, respectively. The advantage of updating the parameters throughout the forecast is that it increases the amount of random variability in the parameters, and increases the volume of parameter values explored during the period of the forecast. However, a disadvantage of this method is that perturbing the parameters during the forecast may introduce jumps, loss of conservation of quantites that should be conserved, and changes of state, which may lead to undesirable effects on the forecast. For the purposes of this study, the RP scheme is a logical choice since the basic RP framework already exists in the Met Office Unified Model (MetUM), and some parameters for which there is genuine uncertainty in their values had already been identified and their uncertainty quantified. We note that this method of rep- resenting model error is not guaranteed to increase the ensemble spread, due to the nonlinear dependence of the model on the parameters.

In Sect. 2 we describe the configuration of the MetUM, the formulation of the RP scheme and the evaluation tools used, and give an outline of the experiments performed in Sects. 4 and 5. In Sect. 3 we describe the meteorology of the case used in this study, and show results of parameter sensitivity tests performed for this case. In Sect. 4 we evaluate the performance of the control (no model error) ensemble, and in Sect. 5 we evaluate the effects of model error variability on the forecast skill and on the spread of the ensemble. Finally, Sect. 6 provides a summary and discussion of our results.

\section{Methodology}

\subsection{Description of the $1.5 \mathrm{~km}$ EPS}

This work was performed using the MetUM version 7.8. The MetUM is a finite-difference model that solves the nonhydrostatic, fully compressible, deep-atmosphere dynamical equations with a semi-Lagrangian, semi-implicit integration scheme. The equations are solved on a horizontally staggered Arakawa $\mathrm{C}$ grid and a terrain-following hybridheight vertical coordinate with Charney-Phillips grid staggering (Davies et al., 2005). In all the MetUM ensemble systems discussed here, boundary-layer mixing is parameterised using the scheme of Lock et al. (2000) and large-scale precipitation is parameterised using the scheme of Wilson and Ballard (1999). In the global and regional formulations of the ensemble system (MOGREPS-G and MOGREPS-R, respectively, described in more detail below), convection is parameterised using the scheme of Gregory and Rowntree (1990); no convection scheme is used in the $1.5 \mathrm{~km}$ EPS. To limit the occurrence of grid-point convection in the $1.5 \mathrm{~km}$ EPS, a prognostic rain variable is advected with the wind field, following Sect. 2d of Lean et al. (2008). In MOGREPS-G and MOGREPS-R, gravity-wave drag is parameterised using the scheme of Webster et al. (2003); this scheme is not active in the $1.5 \mathrm{~km}$ EPS.

The $1.5 \mathrm{~km}$ EPS has a horizontal grid spacing of $1.5 \mathrm{~km}$, with $360 \times 288$ grid points covering a domain over the southern UK (Fig. 1). The model has 70 vertical levels with variable spacing, with the highest vertical resolution in the boundary-layer, and a model lid at around $38 \mathrm{~km}$. This model is run with a time step of $50 \mathrm{~s}$. The $1.5 \mathrm{~km}$ EPS is nested within MOGREPS-R, which has a domain covering the North Atlantic and Europe and a horizontal resolution equivalent to $18 \mathrm{~km}$ in the mid-latitudes and 70 vertical levels, with a model lid at around $80 \mathrm{~km}$. MOGREPS-R is nested within MOGREPS-G, which covers the full globe and runs with a 
resolution equivalent to $60 \mathrm{~km}$ in the mid-latitudes and the same vertical levels as MOGREPS- $\mathrm{R}^{1}$.

A 24-member ensemble is generated in MOGREPS-G using an ensemble transform Kalman filter (ETKF) (Bishop et al., 2001). The ETKF is used to produce a set of perturbations which can be added to the Met Office 4-D-Var analysis to give the initial conditions (ICs) for the ensemble members. A detailed description of the way that the ETKF is used to generate these IC perturbations is given in Bowler et al. (2008). MOGREPS-G is run with $12 \mathrm{~h}$ cycling ${ }^{2}$, starting at 00:00 UTC and 12:00 UTC. MOGREPS-R also runs with $12 \mathrm{~h}$ cycling starting at 06:00 UTC and 18:00 UTC, but takes its initial conditions and hourly lateral boundary conditions (LBCs) from MOGREPS-G. In this study we run a MOGREPS-G forecast starting at 00:00 UTC 20 September 2011 and extend the forecast to 19:00 UTC. We use this to provide ICs and LBCs for a MOGREPS-R forecast starting at 06:00 UTC, which outputs hourly ICs and LBCs for the 1.5 km EPS from 07:00 UTC to 18:00 UTC.

The $1.5 \mathrm{~km}$ EPS is described in detail by Migliorini et al. (2011). Subsequent improvements to the $1.5 \mathrm{~km}$ EPS setup using the so-called scale-selective ETKF to avoid ensemble perturbation mismatches between the ICs and LBCs are described by Caron (2013). In this study we run a $13 \mathrm{~h}$ forecast with hourly ETKF cycling for the first $6 \mathrm{~h}$, followed by a $7 \mathrm{~h}$ forecast, forced by hourly LBCs from MOGREPS-R. The system is described schematically in Fig. 2. In each of the ETKF cycles, the initial conditions for the $1.5 \mathrm{~km}$ EPS control member are produced from a 3-D-Var analysis, with LBCs provided by a $4 \mathrm{~km}$ grid-spacing model with a domain covering the whole UK. For each $1.5 \mathrm{~km}$ EPS ensemble member, a perturbation is applied to the control member ICs. These IC perturbations are derived from MOGREPS$\mathrm{R}$ as follows: in the first cycle, the IC perturbations for the $1.5 \mathrm{~km}$ EPS ensemble members are downscaled from the MOGREPS-R IC perturbations; in subsequent cycles, the $1.5 \mathrm{~km}$ EPS IC perturbations are partitioned into small-scale and large-scale components. The small-scale component of the IC perturbations is derived by applying the scale-selective ETKF to the $1.5 \mathrm{~km}$ EPS forecast perturbations from the previous cycle. The large-scale component of the $1.5 \mathrm{~km}$ EPS ICs is derived by using the downscaled MOGREPS-R forecast perturbations for each ensemble member to perturb the $1.5 \mathrm{~km}$ analysis. An inflation factor is applied to scale the perturbations. This ensures that the innovation variance is consistent with observations at $T+1$ of the forecast (Migliorini et al., 2011; Caron, 2013). A spatially and temporally fixed inflation factor value is used throughout the forecast. A suitable value for the inflation factor in the particular case studied here was determined by examining power spectra of po-

\footnotetext{
${ }^{1}$ The current operational version of MOGREPS-G runs at a higher resolution than this, with a grid length of $33 \mathrm{~km}$ in the midlatitudes.

${ }^{2}$ The current operational version has $6 \mathrm{~h}$ cycling.
}

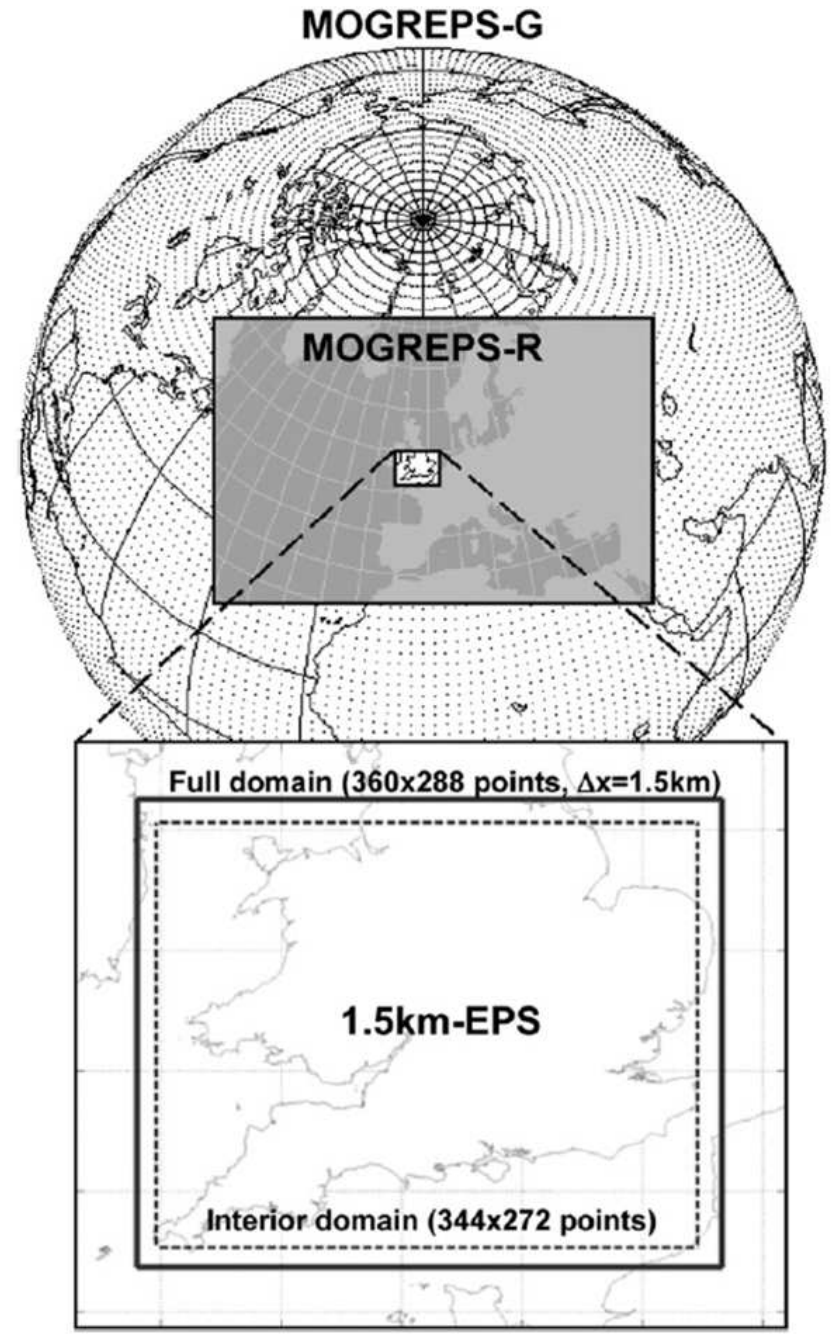

Fig. 1. Schematic diagram showing the model domains of MOGREPS-G, MOGREPS-R and the $1.5 \mathrm{~km}$ EPS used here. Taken from Fig. 1 of Caron (2013). ${ }^{\circ}$ Crown Copyright 2013, Met Office.

tential temperature and horizontal wind using data from four different model levels, as was done by Caron (2013). The IC perturbations and analysis increments are added to the background forecast gradually over a one hour period $(T-30 \mathrm{~min}$ to $T+30 \mathrm{~min}$ ) using the Incremental Analysis Update (IAU) scheme. Hourly LBCs for the control member are derived from the MOGREPS-R control member. Perturbed LBCs are provided for each ensemble member, again by MOGREPSR. During the ETKF cycles, the perturbed LBCs are introduced using the incremental LBC update (ILBCU) method (Caron, 2013) to gradually apply the corresponding LBC perturbations over the same one-hour time period as the IAU scheme acts. 


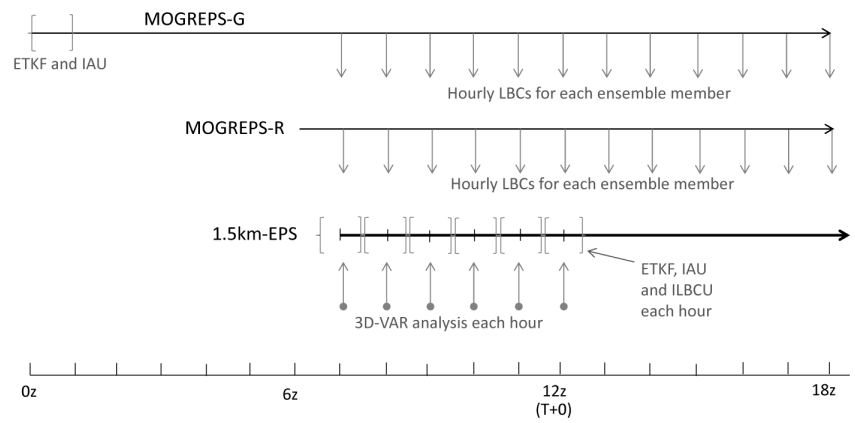

Fig. 2. Schematic diagram showing the model setup of the $1.5 \mathrm{~km}$ EPS used in this study. Times shown are for 20 September 2011.

\subsection{The random parameters scheme as a method of representing model error}

The RP scheme is used operationally in MOGREPS (Bowler et al., 2008). The scheme is designed to simulate model error due to uncertainty in the parameterisation of sub-grid scale processes. The RP scheme takes a set of parameters from various parameterisation schemes and treats them as stochastic variables. For each parameter, a physically sensible range of values has been defined as advised by experts. The parameter is allowed to vary within this range.

In the original RP scheme, two parameters from each of the boundary layer, large-scale precipitation, gravity-wave drag and convection schemes were selected (see Table 1 of Bowler et al. (2008) for the full list of parameters and values). Five additional boundary layer parameters were later added to the RP scheme following the work of Zadra and Lock (2010). The full set of boundary layer and large-scale precipitation scheme parameters used in this updated scheme is shown in the top part of Table 1.

The parameter values are calculated first using the firstorder autoregression model

$P_{t}^{0}=\mu+r\left(P_{t-1}-\mu\right)+\epsilon$,

where $P_{t}^{0}$ and $P_{t-1}$ are the intermediate and previous parameter values, $\mu$ is the mean value ${ }^{3}$ of the parameter distribution (taken to be the default value of that parameter) and $r$ is the auto-correlation constant (set as $r=0.95$ ). $\epsilon$ is the stochastic shock term, sampled from a uniform distribution in the range $\pm\left(P_{\max }-P_{\min }\right) / 3$, where $P_{\max }$ and $P_{\min }$ are the maximum and minimum values for the parameter. If $P_{t}^{0}$ is outside of the prescribed range, it is rounded down or up to give the new parameter value, $P_{t}$ :

$P_{t}= \begin{cases}P_{\min } & \text { if } P_{t}^{0}<P_{\min }, \\ P_{\max } & \text { if } P_{t}^{0}>P_{\max }, \\ P_{t}^{0} & \text { otherwise. }\end{cases}$

\footnotetext{
${ }^{3} \mu$ is not necessarily the true mean of the resulting distribution of parameter values. The true mean is influenced by the rounding process described in Eq. (2).
}

(a)

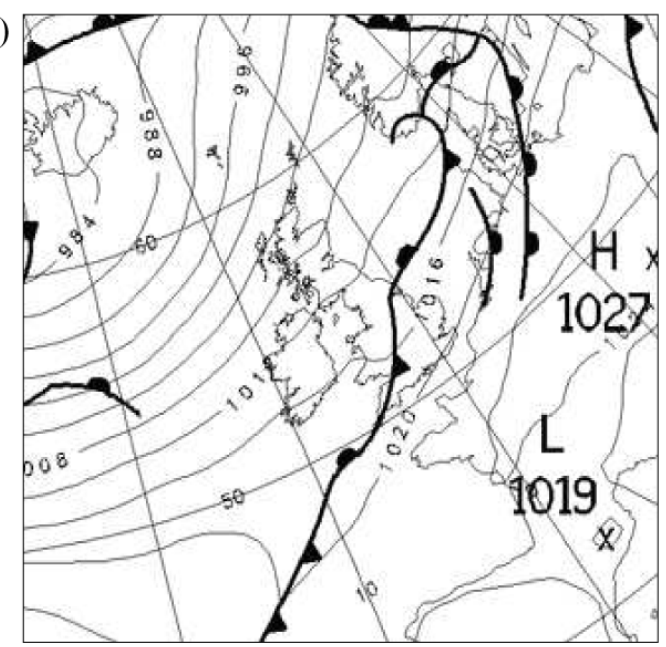

(b)

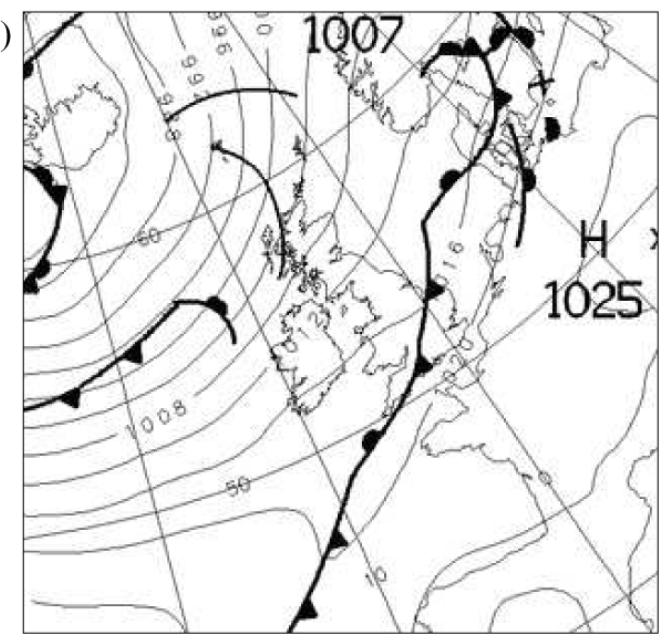

Fig. 3. Met Office mean sea level pressure analysis charts valid at (a) 12:00 UTC and (b) 18:00 UTC 20 September 2011. Grey pressure contours every $4 \mathrm{hPa} .{ }^{\odot}$ Crown Copyright 2011, Met Office.

The range of $\epsilon$ and the value of $r$ were chosen following a series of $72 \mathrm{~h}$ forecasts and selecting the combination of values that gave the maximum spread and best deterministic scores for individual ensemble members (Bowler et al., 2008). These values are therefore tuned for the resolution of MOGREPS-G, which at the time had $90 \mathrm{~km}$ grid spacing.

In the standard formulation of the RP scheme, the parameters are updated using Eqs. (1) and (2) once every $3 \mathrm{~h}$. The parameter values are then fixed for a period of $3 \mathrm{~h}$ until the next update time. The same parameter values are used for all grid points. Each time the scheme is applied, a new random number $k \in[0,1]$ is generated to give a different value of $\epsilon$ defined as $\epsilon=(2 k-1)\left(P_{\max }-P_{\min }\right) / 3$. A different random number is used for each parameter so that they vary independently from one another. On the first application of the RP scheme, the parameter values are set to random values within the parameter range. Each ensemble member, including the 
Table 1. Parameters used in the modified RP scheme. The four pairs of parameters that vary together (using the same random number to update $\epsilon$ in Eq. 1) are shown in their respective pairs in italics and bold. Parameter $R i_{\mathrm{c}}$ varies as $R i_{\mathrm{c}}=10 / g_{0}$.

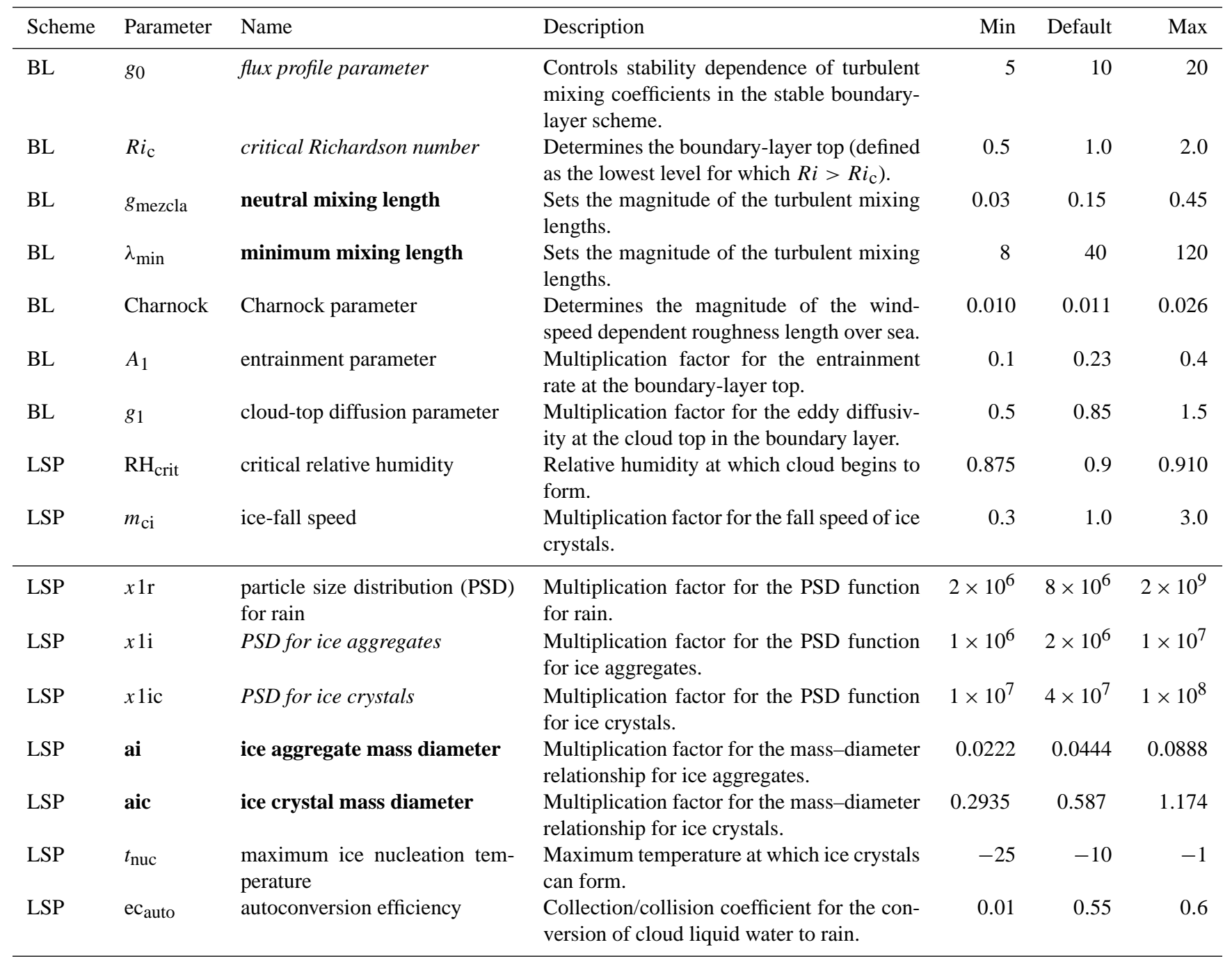

control member, is given a different set of random parameter perturbations by the RP scheme.

\subsection{Modifications to the scheme for the convective-scale EPS}

To apply the RP scheme to a forecast ensemble run in the $1.5 \mathrm{~km}$ EPS convective-scale setup, it was necessary to make some modifications to the existing scheme. Importantly, at this resolution, the convection scheme and gravity-wave drag schemes are not used. Therefore, only parameters in the boundary layer and large-scale precipitation schemes were perturbed. Sensitivity tests were used to determine appropriate parameters in the large-scale precipitation scheme to perturb. These tests are described in Sect. 3.3. The full set of parameters used here is given in Table 1 .
Given the shorter time step of the high-resolution forecast compared with the global and NAE forecasts (50 s compared with 5-10 min) and the short forecast lead times we are interested in, it was appropriate to revise the time interval between calls to the RP scheme ( $3 \mathrm{~h}$ in the original scheme). We test update times of 30 and $60 \mathrm{~min}$, which were chosen to allow the parameters to vary smoothly over time, without giving continual shocks to the model. Consideration was also given to the timescales of the processes that the parameterisation schemes represent. A further version of the RP scheme, holding parameters fixed throughout the forecasts, was also used. In all cases the first application of the RP scheme is at $T+30 \mathrm{~min}$, after the IAU and ILBCU schemes have finished. This is to allow the IC and LBC perturbations to be added completely before the RP scheme is applied. 


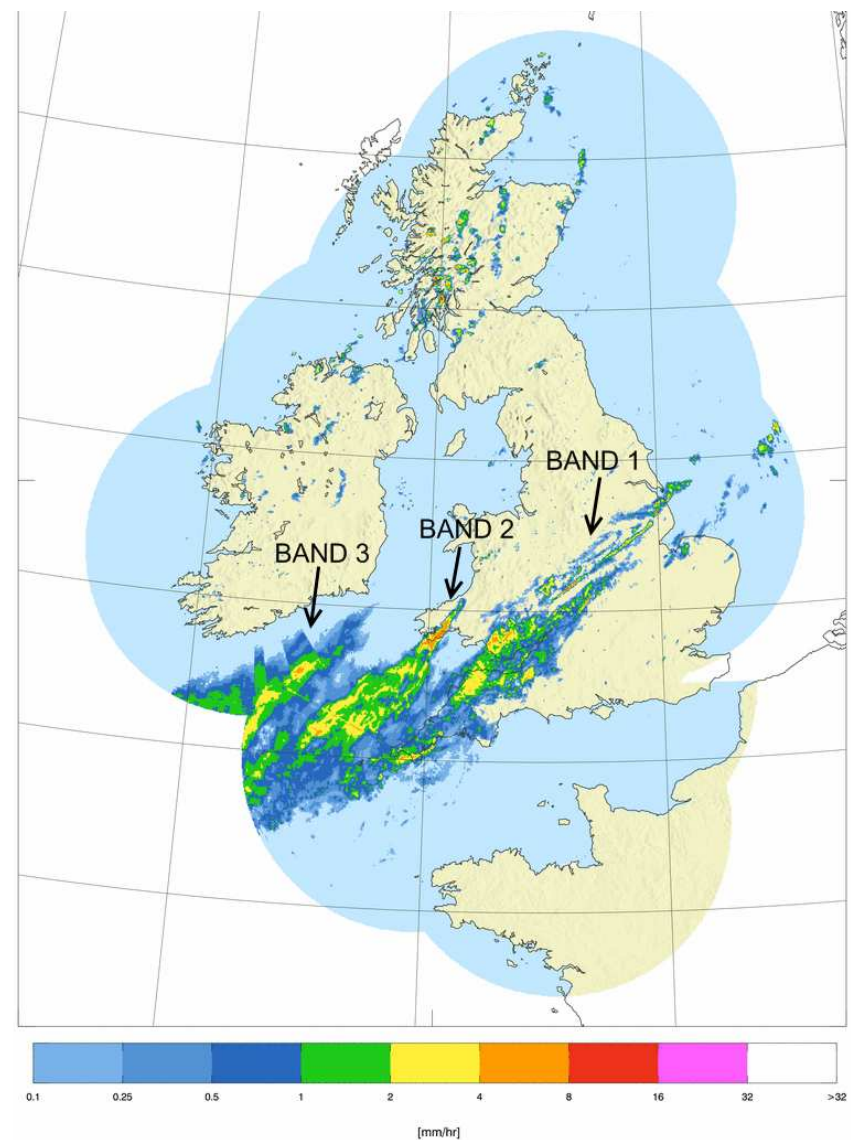

Fig. 4. Radar-derived surface precipitation rate ( $1 \mathrm{~km}$ composite) at 15:00 UTC 20 September 2011. ${ }^{\circledR}$ Crown Copyright 2011, Met Office.

\subsection{Evaluation and verification methods}

We evaluate the spread and skill of the ensemble by considering near-surface fields $(1.5 \mathrm{~m}$ temperature, $1.5 \mathrm{~m}$ relative humidity $(\mathrm{RH})$ and $10 \mathrm{~m}$ wind speed), rainfall rate and rainfall accumulation. These quantities were chosen because they could be evaluated against surface and radar observations that were available to us.

To evaluate the spread of the ensemble we use two different diagnostics: the first is a domain-averaged measure of the ensemble spread at each time; the second, for the hourly rain accumulation only, is the correspondence ratio (CR), which gives a measure of spread for a whole field rather than individual grid points (as used by Gebhardt et al., 2011). We define the spread for an $n$-member ensemble as

$\operatorname{spread}=\frac{1}{n x \times n y} \sum_{i, j=1}^{n x, n y} \sigma_{i, j}$, where

$\sigma_{i, j}=\sqrt{\frac{1}{n-1} \sum_{k=1}^{n}\left(p_{i, j, k}-\overline{p_{i, j}}\right)^{2}}$,

$n x$ and $n y$ are the numbers of grid points in the $x$ and $y$ directions, respectively (in our case $n x=360$ and $n y=288$ ), $p_{i, j, k}$ denotes the value of field $p$ at point $(i, j)$ for ensemble member $k$ and $\overline{p_{i, j}}$ is the ensemble mean value of field $p$ at point $(i, j)$. CR is calculated following Gebhardt et al. (2011) as $\mathrm{CR}=N\left(P_{\text {all }}\right) / N\left(P_{\geq 1}\right)$, where $N\left(P_{\text {all }}\right)$ is the number of grid points where all members forecast an event, and $N\left(P_{\geq 1}\right)$ is the number of grid points where at least one member forecasts the event. A low CR indicates large ensemble spread, while a high $\mathrm{CR}$ value indicates that the ensemble members are generally in agreement.

To evaluate the forecast skill of the ensemble by comparing with observations, we use three methods: the first, for rain and near-surface fields, is the continuous ranked probability score (CRPS); the second, for rainfall accumulation only, is the precipitation skill score (PSS); the third, for rainfall rate only, is the innovation magnitude.

The CRPS is a way of measuring the closeness between two cumulative distribution functions (CDFs). In our case the two CDFs are those of a forecast, $F_{\mathrm{f}}(x)$, and of an observation, $F_{\mathrm{o}}(x)$. The definition of the CRPS at point $(i, j)$ and for quantity $x_{i, j}$ is the total squared difference between these CDFs:

$\operatorname{CRPS}_{i, j}=\int_{x_{i, j}=-\infty}^{\infty} \mathrm{d} x_{i, j}\left(F_{\mathrm{f}}\left(x_{i, j}\right)-F_{\mathrm{o}}\left(x_{i, j}\right)\right)^{2}$,

(Wilks, 2006), where the CDFs are $F_{\mathrm{f} / \mathrm{o}}\left(x_{i, j}\right)=$ $\int_{x_{i, j}^{\prime}=-\infty}^{x_{i, j}} \mathrm{~d} x_{i, j}^{\prime} P_{\mathrm{f} / \mathrm{o}}\left(x_{i, j}^{\prime}\right)$ and where $P_{\mathrm{f} / \mathrm{o}}\left(x_{i, j}\right)$ is the probability density function (PDF) for the forecast/observation at the point $(i, j)$. The forecast's PDF is described by the ensemble members, $x_{i, j}^{(k)}$, by assuming that each is equally likely: $P_{\mathrm{f}}\left(x_{i, j}\right)=\sum_{i=1}^{N} \delta\left(x_{i, j}-x_{i, j}^{(k)}\right)$, where $\delta$ is the Dirac delta-function. In previous works using the CRPS, an observation's PDF assumes that the observation is perfect, i.e. that $P_{\mathrm{o}}\left(x_{i, j}\right)=\delta\left(x_{i, j}-y_{i, j}^{\mathrm{o}}\right)$, where $y_{i, j}^{\mathrm{o}}$ is the observed value, which leads to the observation's CDF being a Heaviside step function. This simplifies the calculation of the CRPS as it avoids the need to do explicit quadrature (see Sect. 4 of Hersbach, 2000). In this work though we account for imperfect observations by assuming that each observation's PDFs is a normal distribution with finite width, $\sigma_{\mathrm{o}}$. In this case the CRPS reduces to the following calculation:

$$
\begin{aligned}
& \operatorname{CRPS}_{i, j}=\int_{x_{i, j}=-\infty}^{\infty} \mathrm{d} x_{i, j}\left\{\frac{1}{N} n_{\mathrm{f}}\left(x_{i, j}\right)-\right. \\
& \left.\frac{1}{2}\left[1+\operatorname{erf}\left(\frac{x_{i, j}-y_{i, j}^{\mathrm{o}}}{\sqrt{2} \sigma_{\mathrm{o}}}\right)\right]\right\}^{2},
\end{aligned}
$$



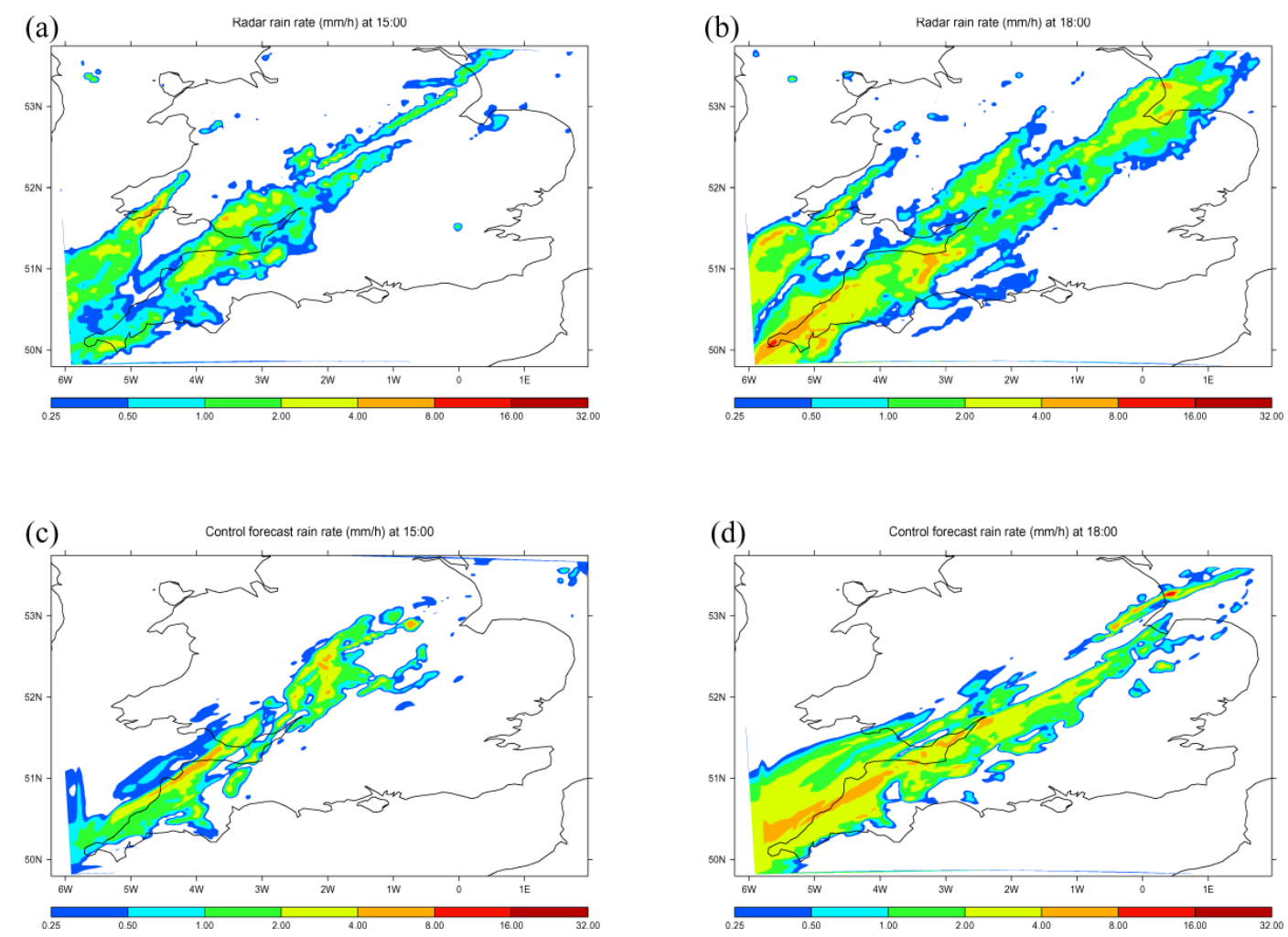

Fig. 5. Observed and model rain rate $\left(\mathrm{mm} \mathrm{h}^{-1}\right)$. Left column: 15:00 UTC $(T+3)$, right column: 18:00 UTC $(T+6) 20$ September 2011. Top row: radar-derived surface precipitation rates $(1 \mathrm{~km}$ composite), bottom row: control forecast rainfall rate at the surface.

where $n_{\mathrm{f}}\left(x_{i, j}\right)$ is the number of ensemble members whose forecast values are less than or equal to $x_{i, j}$ and $\operatorname{erf}(x)$ is the standard error function $\operatorname{erf}(x)=\frac{2}{\sqrt{\pi}} \int_{x^{\prime}=0}^{x} \exp -x^{2} \mathrm{~d} x$. Numerical quadrature is necessary to evaluate this integral. Note that a lower CRPS indicates a more skillful forecast.

To calculate the PSS, the hourly rainfall accumulation data for the model forecast and radar observations are first resampled to $13.5 \mathrm{~km}$. This reduces the chance of penalising any small displacement errors twice, which can happen in high resolution forecasts if using grid point comparison methods. A similar resampling was done by Caron (2013) (to $15 \mathrm{~km}$ ) when using the PSS. Next, the Brier score (BS, Wilks (2006)) is calculated, and is defined as

$\mathrm{BS}=\frac{1}{n x \times n y} \sum_{i, j=1}^{n x, n y}\left(f_{i, j}-o_{i, j}\right)^{2}$,

where $f_{i, j}$ is the probability of a given event occurring in the model ensemble data, and $o_{i, j}$ is a binary indicator of the occurrence of the event in the observations. In this case the event is the exceedance of a threshold rain rate at a given grid point. The PSS for an ensemble ENS is given by

$\mathrm{PSS}=1-\frac{\mathrm{BS}_{\mathrm{ENS}}}{\mathrm{BS}_{\mathrm{CTL}}}$.
The PSS is therefore given with respect to a control ensemble, which here is the ensemble with IC and BC perturbations but no RP scheme applied (CTL in Table 2).

To compare the ensemble mean with the radar observations we calculate the innovation magnitude, $d$, defined at each grid point as the absolute difference between the mean value of the ensemble at that point and the observation value at that point:

$d_{i, j}=\left\|\overline{p_{i, j}}-y_{i, j}^{\mathrm{o}}\right\|$.

\subsection{Description of the experiments}

The various ensemble experiments discussed in Sects. 4 and 5 are listed in Table 2. The control ensemble (CTL) is composed of a set of 24 six-hour forecasts with ICs at 12:00 UTC derived from MOGREPS-R IC perturbations and previous cycles of the $1.5 \mathrm{~km}$ EPS, as described in Sect. 2.1. The IC and LBC perturbations are introduced gradually between $T-30$ and $T+30$ using the IAU and ILBCU schemes, respectively. This ensemble has no representation of model error variability. Experiments labelled $\mathrm{IC}+\mathrm{BC}+\mathrm{RP}^{*}$ (where here and throughout this paper, $*$ is shorthand for either "fix", "30" or "60") use the same IC and LBC perturbations as the control ensemble but also use the RP scheme with different lengths of time between parameter updates (once, every $30 \mathrm{~min}$ and every $60 \mathrm{~min}$, respectively), starting from 
(a)

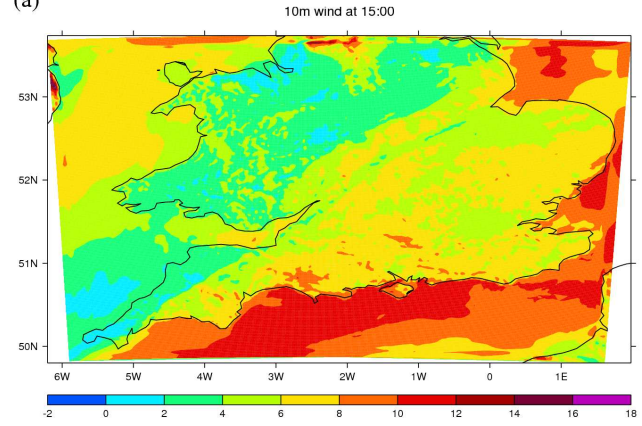

(c)

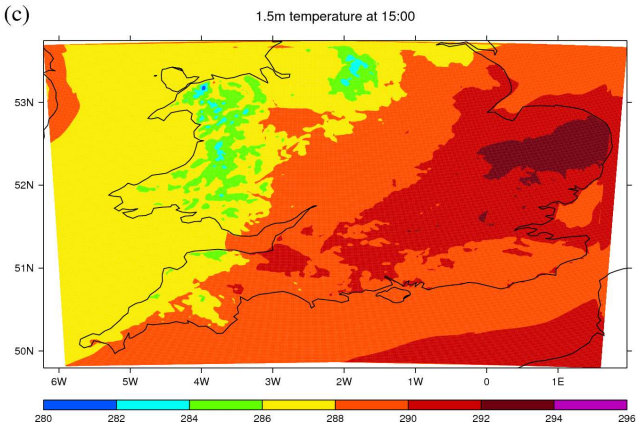

(b) $10 \mathrm{~m}$ wind at 18:00

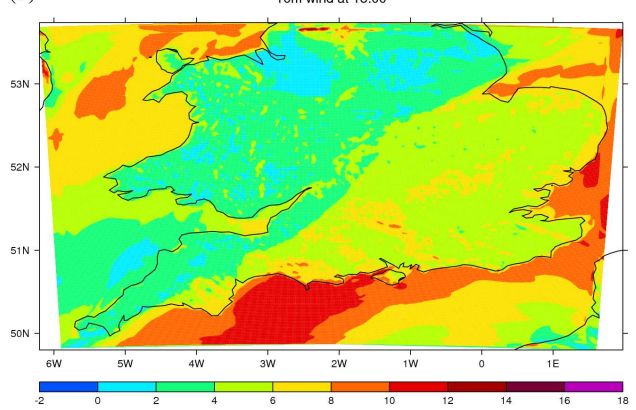

(d)

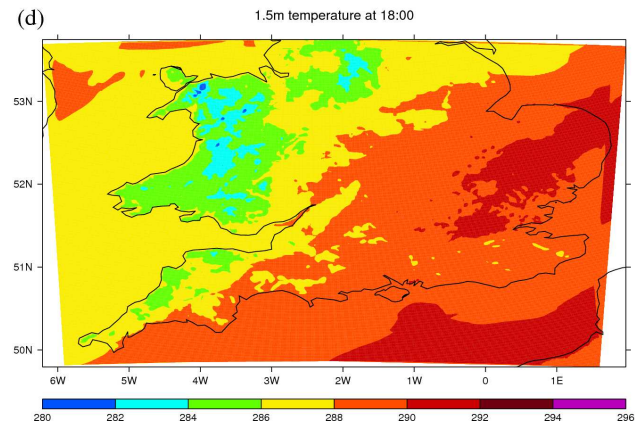

Fig. 6. Control forecast. Left column: 15:00 UTC $(T+3)$, right column: 18:00 UTC $(T+6) 20$ September 2011 . Top row: $10 \mathrm{~m}$ wind speed $\left(\mathrm{m} \mathrm{s}^{-1}\right)$, bottom row: $1.5 \mathrm{~m}$ temperature $(\mathrm{K})$.

Table 2. Summary of model configurations for the ensemble experiments.

\begin{tabular}{llcc}
\hline Ensemble name & Description & IC and LBC perts & RP scheme \\
\hline CTL & Control (perturbed ICs and LBCs, no RP scheme) & Yes & No \\
IC + BC + RPfix & perturbed ICs and LBCs, RP scheme applied once & Yes & Yes \\
IC + BC + RP30 & perturbed ICs and LBCs, RP scheme applied every 30 min & Yes & Yes \\
IC + BC + RP60 & perturbed ICs and LBCs, RP scheme applied every 60 min & Yes & Yes \\
RPfix & RP scheme applied once & No & Yes \\
RP30 & RP scheme applied every 30 min & No & Yes \\
RP60 & RP scheme applied every 60 min & No & Yes \\
\hline
\end{tabular}

$T+30$ min (after the IAU and ILBCU have finished). Experiments labelled RP* have no IC and LBC perturbations: their members all use the unperturbed ICs and LBCs of the control member, with model error represented by applying the RP scheme. These ensembles therefore show the effects of using the RP scheme to perturb parameters, without the effects of perturbing the ICs and LBCs.

\section{Description of the case and sensitivity tests to inform the modifications to the RP scheme}

\subsection{Overview}

For this study we focus on a case on 20 September 2011 characterised by the passage of a cold front over the south- ern UK (Fig. 3). It can be seen from the analysis charts that this is part of a long, trailing front with several weak frontal waves. This frontal structure passed across the southern UK in a north-eastward direction. There are two main reasons for selecting this particular case. First, it occurred during a three-week field campaign period of the DIAMET (DIAbatic influences on Mesoscale structures in ExTratropical storms) project (funded by the Natural Environment Research Council (NERC)), and was thus selected as a DIAMET intensive observation period (IOP-2). We therefore have access to high-resolution observations for this case, in addition to those made operationally, including in situ aircraft measurements, dropsonde profiles and extra radiosonde ascents from some UK stations. These non-operational observations are not used in the study reported in this paper, but are being 


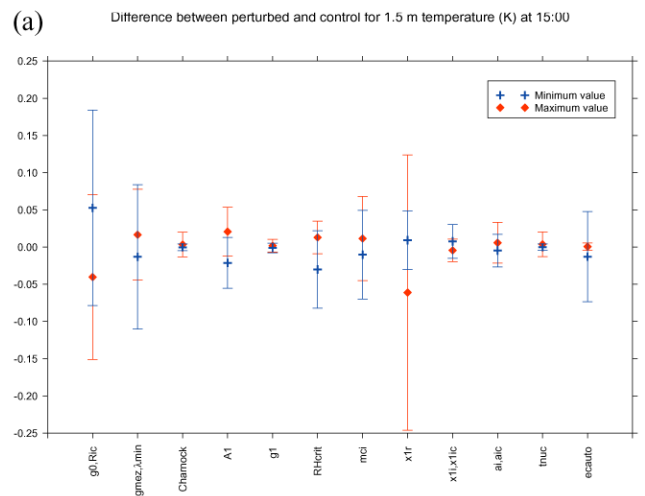

(c) RMSE of perturbed relative to control for $1.5 \mathrm{~m}$ temperature $(K)$ at $15: 00$

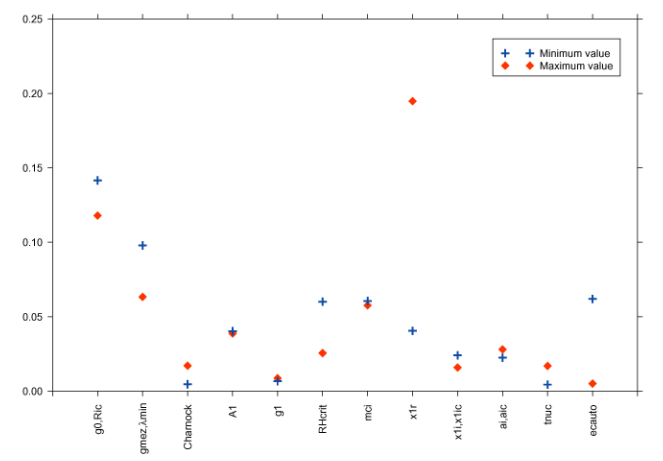

(b) Ditterence between perturbed and control tor $10 \mathrm{~m} \mathrm{u}-$-wind $(\mathrm{m} / \mathrm{s})$ at 15:00

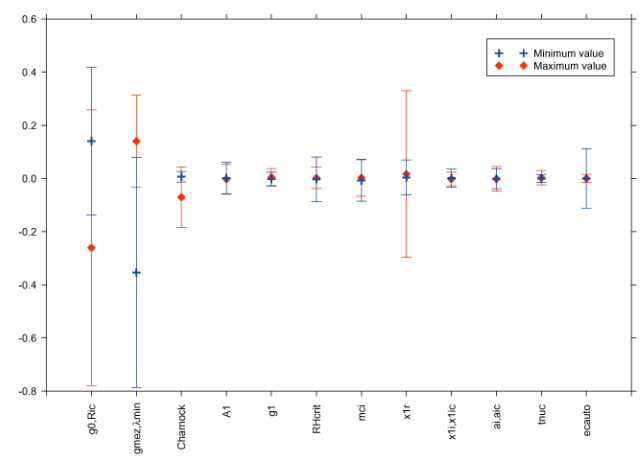

(d) RMSE of perturbed relative to control for $10 \mathrm{~m}$ u-wind $(\mathrm{m} / \mathrm{s})$ at 15:00

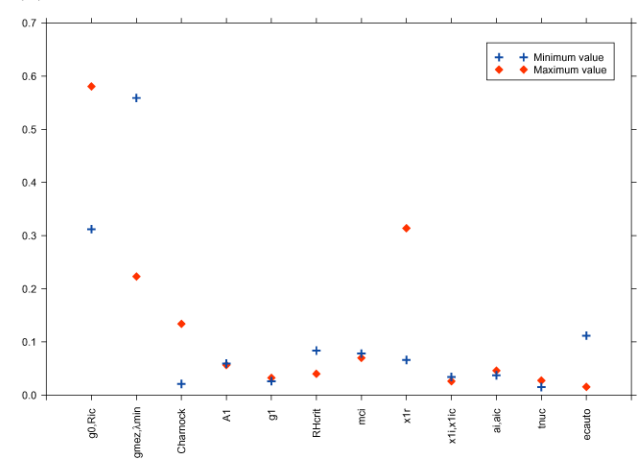

Fig. 7. Sensitivities of the forecasts at $T+3$ (15:00 UTC) to perturbing parameters to their maximum and minimum values. Top row: difference between perturbed and control forecast at each point, averaged over the domain. Error bars show the standard deviation of the differences. Bottom row: RMS difference between the perturbed and control forecast at each point, averaged over the domain. Left column: $1.5 \mathrm{~m}$ temperature. Right column: $u$ component of $10 \mathrm{~m}$ wind.

used in ongoing related work (Migliorini, Bannister, Rudd and Baker, in preparation). The second reason for choosing this case is that it has an unusual triple banded structure in the rain band passing over the UK, which was present in the radar rain rate (Fig. 4) but was not captured by the Met Office operational high-resolution deterministic forecast (the UKV forecast; $1.5 \mathrm{~km}$ grid length, covering the whole UK; not shown). This triple rain band feature, and the fact that it was not captured by the operational deterministic forecast, makes it an interesting case to study using an ensemble of forecasts. It is not clear from either the model forecasts or the radar observations what the cause of this banded structure is; this is the subject of ongoing work and is beyond the scope of this paper.

We focus on the period 12:00 UTC to 18:00 UTC, and are particularly interested in evaluating the performance and spread of the ensemble at 15:00 UTC $(T+3)$ as this is typically a useful timescale for convective scale forecasts. This should also allow time for model error introduced by the RP scheme to have effect before information coming through the boundaries begins to dominate (Gebhardt et al., 2011).

\subsection{Control forecast from the $1.5 \mathrm{~km}$ EPS}

The control forecast (using the standard default parameter values in each of the parameterisation schemes) captures the main rain band (Fig. 5c and d) but does not capture the second band over South Wales seen in the radar (Fig. 5a and b). Note that during the period studied here the third rain band in Fig. 4 does not reach the $1.5 \mathrm{~km}$ EPS domain. There is some smaller-scale banding along the rain band in the control forecast at both times shown, indicating that the model is representing the correct type of smaller-scale structure within the rain band. Figure $6 a$ and $b$ show stronger winds ahead of the front than behind the front, with a sharp drop in $10 \mathrm{~m}$ wind speeds over land compared with the wind speeds over the sea, which is likely to be caused by the different surface roughness and orographic effects. The front is relatively weak, with a temperature gradient of around $6 \mathrm{~K}$ across the front (Fig. $6 \mathrm{c}$ and d). The location of the strongest temperature gradient in Fig. $6 \mathrm{~d}$ is consistent with the position of the analysed surface front in Fig. 3b. 

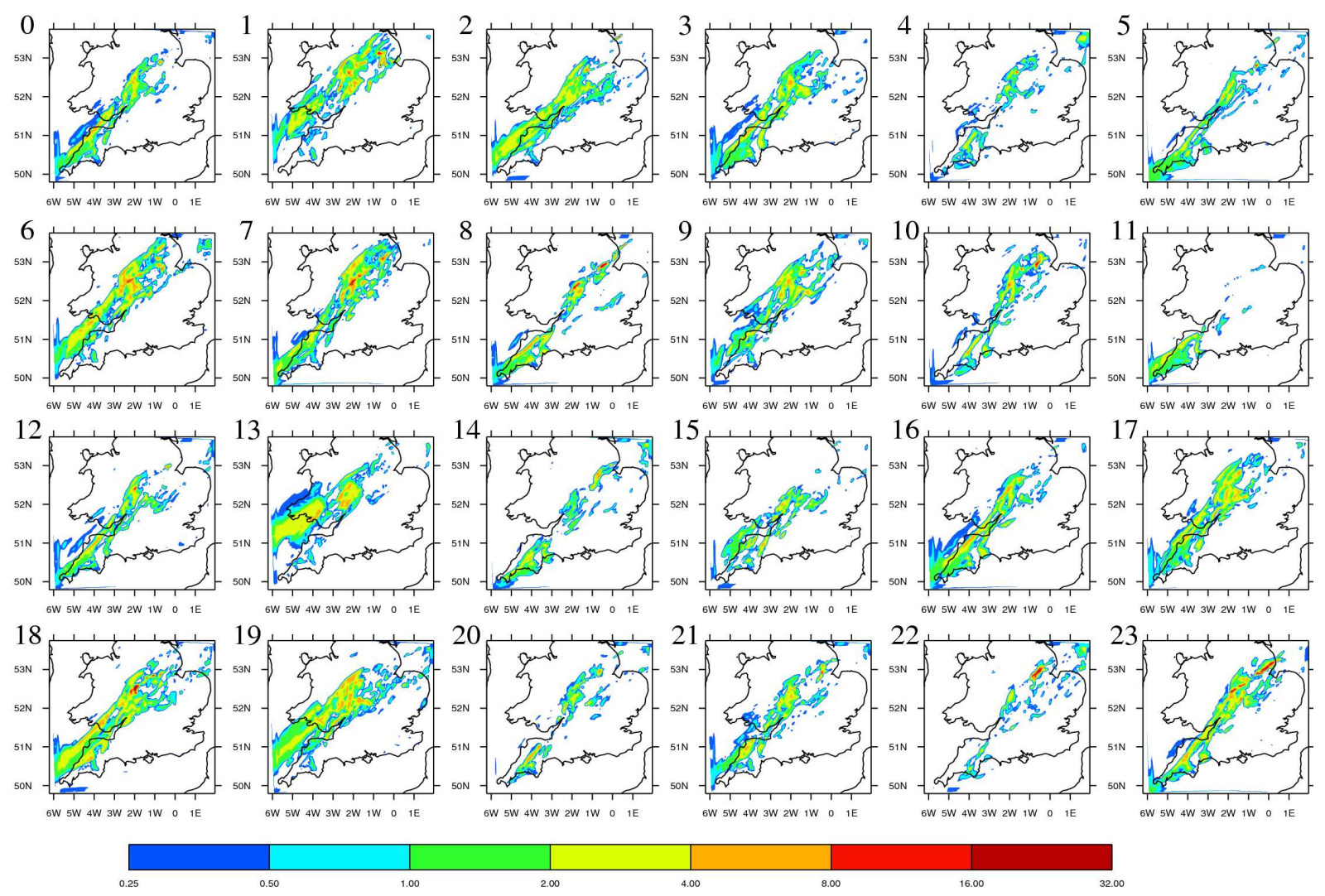

2.00 Rain rate (mm

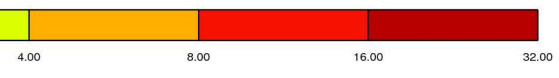

Fig. 8. Control ensemble rainfall rate $\left(\mathrm{mm} \mathrm{h}^{-1}\right)$ at 15:00 UTC. The control member (member 0) is in the top left-hand corner.

\subsection{Parameter sensitivity tests}

In order to make the RP scheme suitable to be used in the $1.5 \mathrm{~km}$ EPS, we first identified suitable parameters to be perturbed within the scheme, in addition to those used in the operational scheme. Since work had already been done by Zadra and Lock (2010) to identify extra parameters to be perturbed in the boundary layer scheme after the RP scheme was originally developed, we focus here on parameters in the large-scale precipitation scheme. An extensive set of around 50 parameters known to have some uncertainty was determined by consulting with experts at the Met Office. For each parameter a suitable range of values was chosen such that it was physically sensible while also reflecting the uncertainty in the value of the parameter.

From this extensive list of parameters, a smaller subset was chosen (Table 1). The first nine parameters listed in Table 1 are those used in the operational RP scheme, although some of the ranges have been changed, based on advice by experts. The last seven parameters in Table 1 are those that we have added to the RP scheme. The selection of these parameters was motivated by choosing from the 50 those with the highest sensitivity, and choosing one parameter for each physical process, in order to minimise the possibility of parameters counteracting each other. Choosing a set of parameters that collectively control a variety of different processes also has the advantage that the overall effects of the scheme may be less case-dependent. In addition, some parameters were chosen to vary together in pairs. These pairs of related parameters are indicated in Table 1 . Parameters $R i_{\mathrm{c}}$ and $g_{0}$ are related by $R i_{\mathrm{c}}=10 / g_{0}$; for the other parameter pairs the same random number is used to update their associated $\epsilon$ in Eq. (1), which ensures that these pairs of parameters remain correlated. In this chosen set of parameters, there are seven parameters from the boundary layer scheme and nine from the large-scale precipitation scheme, but due to the pairings of some parameters there are five independent parameters from the boundary layer scheme and seven independent parameters from the large-scale precipitation scheme.

To test the sensitivity of the forecast to each of these parameters, a series of forecasts was run in which each parameter (or pair of parameters) was perturbed to its maximum or minimum value. The perturbed forecasts were compared with the control forecast to determine the sensitivity of the forecast to each parameter, and also to test in what way the forecast was affected. In particular, it is desirable that perturbing a parameter to its maximum and minimum values gives opposite effects (e.g. one increases the temperature while the other decreases it), than for both cases to have the same result (e.g. a reduction in temperature) which would 

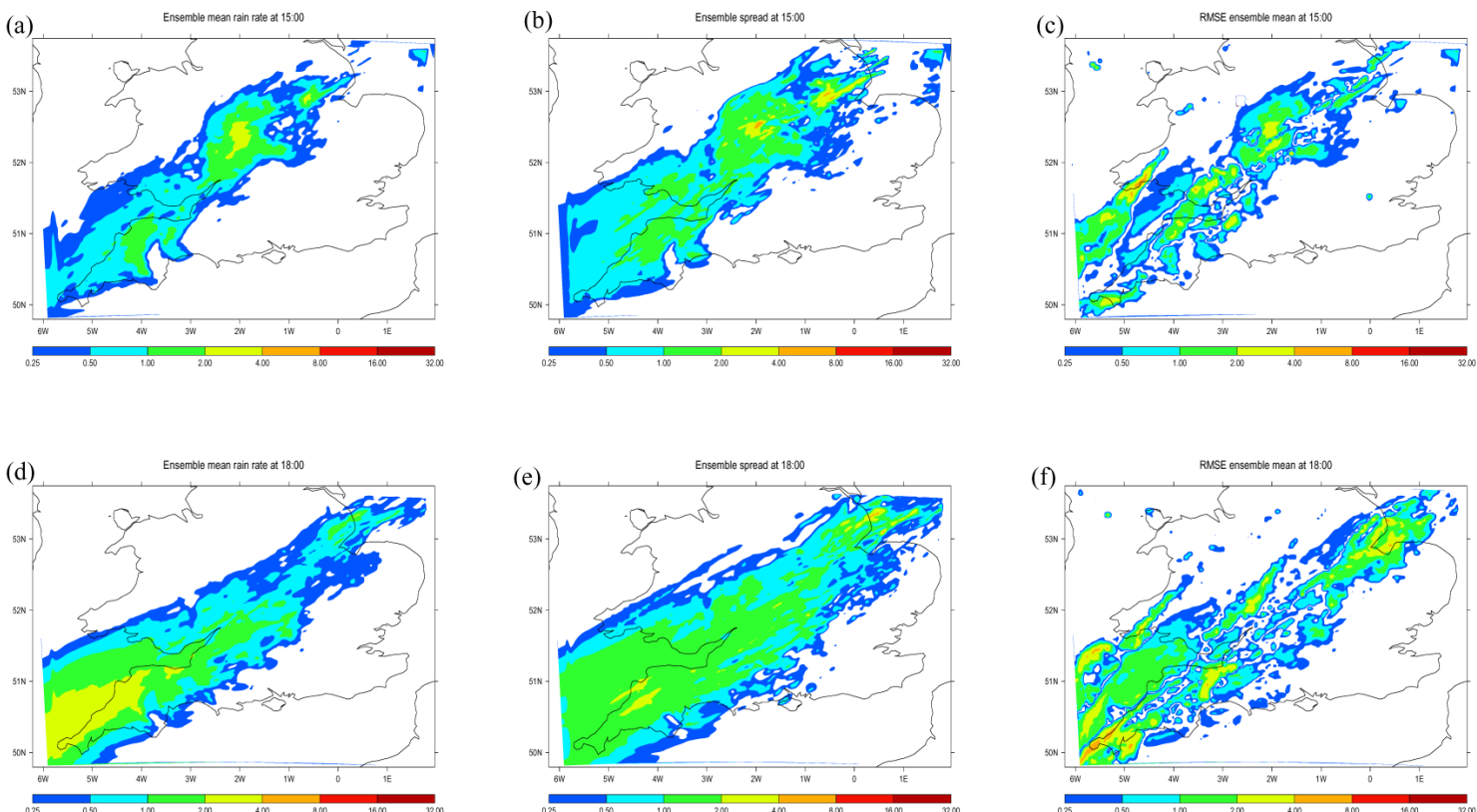

Fig. 9. Spatial distribution of rain rate diagnostics $\left(\mathrm{mm} \mathrm{h}^{-1}\right)$ for the control ensemble. Left column: ensemble mean, middle column: ensemble spread and right column: innovation magnitude of the ensemble mean, at 15:00 UTC (top row) and 18:00 UTC (bottom row) 20 September 2011.

imply that perturbing this parameter randomly may introduce a bias.

The sensitivity of the forecast to perturbing each of the parameters to its maximum and minimum value was examined both by computing domain-averaged differences and rootmean-squared (RMS) differences. Difference maps of surface variables at different forecast lead times were also examined (not shown). These show that different parameters affect the forecast in different parts of the domain. Specifically, the large-scale precipitation parameters have the strongest effects in the region of the rain band, while the effects of boundary layer parameters are generally more widespread, and most strongly affect the north-east part of the domain.

Figure 7 shows the domain-averaged $3 \mathrm{~h}$ sensitivities with respect to each of the 12 independent parameters (or parameter pairs) for $1.5 \mathrm{~m}$ temperature and for the $u$ component of the $10 \mathrm{~m}$ wind. Equivalent plots for $1.5 \mathrm{~m} \mathrm{RH}$ and $v$ component of $10 \mathrm{~m}$ wind (not shown) show qualitatively similar results to those for $1.5 \mathrm{~m}$ temperature and for the $u$ component of $10 \mathrm{~m}$ wind (respectively), in terms of their relative sensitivities to each parameter (although note that the response for $1.5 \mathrm{~m} \mathrm{RH}$ has the opposite sign to the response for $1.5 \mathrm{~m}$ temperature, as might be expected). Figure $7 \mathrm{a}$ and $\mathrm{b}$ show that for all parameters, the domain average effects on the temperature and $\mathrm{u}$-component of wind of perturbing a parameter to its maximum value has an opposite sign to perturbing it to its minimum value. However, the bars showing the standard deviation indicate that there is a large variability in the effect of these parameters over the domain. The RMS difference plots (Fig. 7c and d) give a measure of the magnitude of the sensitivity to each of the parameters. It can be seen that the boundary layer parameter pairs $\left(g_{0}, R i_{\mathrm{c}}\right)$ and $\left(g_{\text {mezcla }}, \lambda_{\min }\right)$, and the large-scale precipitation parameter $x 1 \mathrm{r}$ (perturbed only to its maximum value) have the largest effects on both the temperature and $u$ component of wind. The parameter pairs $\left(g_{0}, R i_{\mathrm{c}}\right)$ and $\left(g_{\text {mezcla }}, \lambda_{\text {min }}\right)$ affect the wind more, while $x 1 r$ has a large impact on the temperature. Although the effects of these parameters dominate in this case, the effects of perturbing the other parameters are not orders of magnitude smaller. The sensitivity to most of the parameters will be dependent on the atmospheric state (in particular the boundary layer stability for the boundary layer parameters, and the cloud amount and type for the large-scale precipitation parameters), and these results confirm that this is a reasonable set of parameters and ranges to choose in order to simulate model error variability.

\section{Evaluation of the control ensemble}

In this section we describe the properties of the control ensemble, CTL, which has IC and LBC perturbations but no representation of model error variability.

All the ensemble members capture the main rain band at 15:00 UTC (Fig. 8), although there is considerable variability in the amount of rainfall associated with this band (compare, for example, members 7 and 11). Inspection of the rain rate in the ensemble members from the driving model, MOGREPS$\mathrm{R}$ (not shown), also shows the main rain band, but no member has the second band, suggesting that the mechanism causing 
(a)

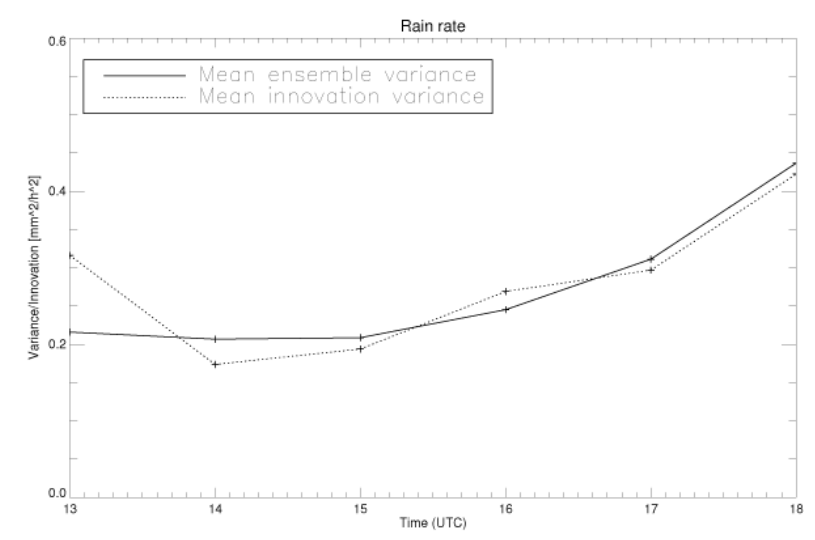

(b)

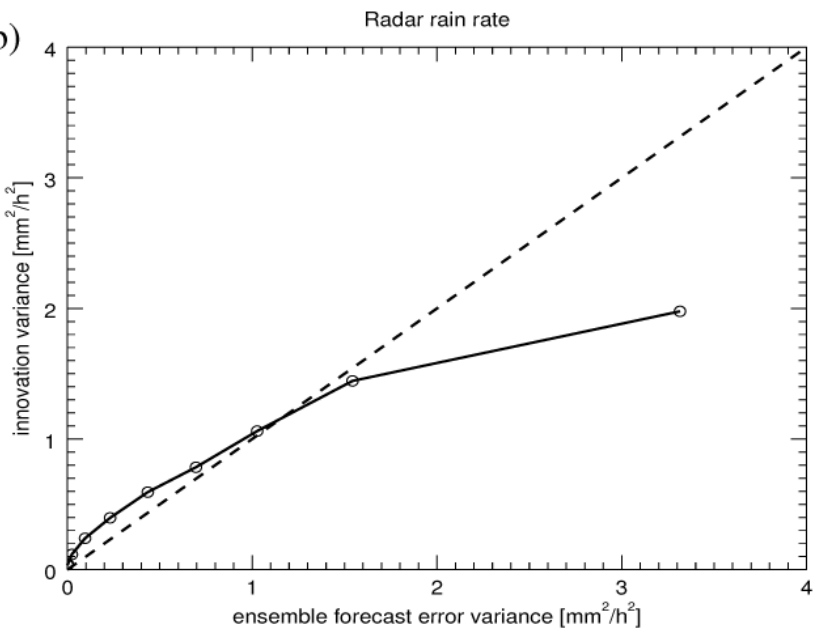

Fig. 10. (a) Domain averaged ensemble variance and innovation variance for rain rate every hour. (b) Spread-skill plot for the control ensemble. In (b) the data for each grid point for each hour of the forecast are binned into 10 bins by ensemble variance.

this banded structure to form in reality was not well represented in MOGREPS-R. Our hope was that the increased resolution might lead to some $1.5 \mathrm{~km}$ EPS members capturing the second band, but Fig. 8 shows that this ensemble also fails to capture the two bands together in any individual member. Whether this is an inevitable consequence of the lack of multiple bands in the driving model is an open question.

There is generally good agreement in the positioning of the main rain band, although three members (members 1, 13 and 19) have the rain band displaced further to the north. This means that, although the two distinct bands seen in the radar are not captured, these three members do have some rain in roughly the location of the second radar rain band. Several of the ensemble members have more intense rain in the northeast part of the rain band, and in particular some of them have one or two localised regions of heavy rain (e.g. members 7,8 and 13) exceeding $16 \mathrm{~mm} \mathrm{~h}^{-1}$, which is not seen in

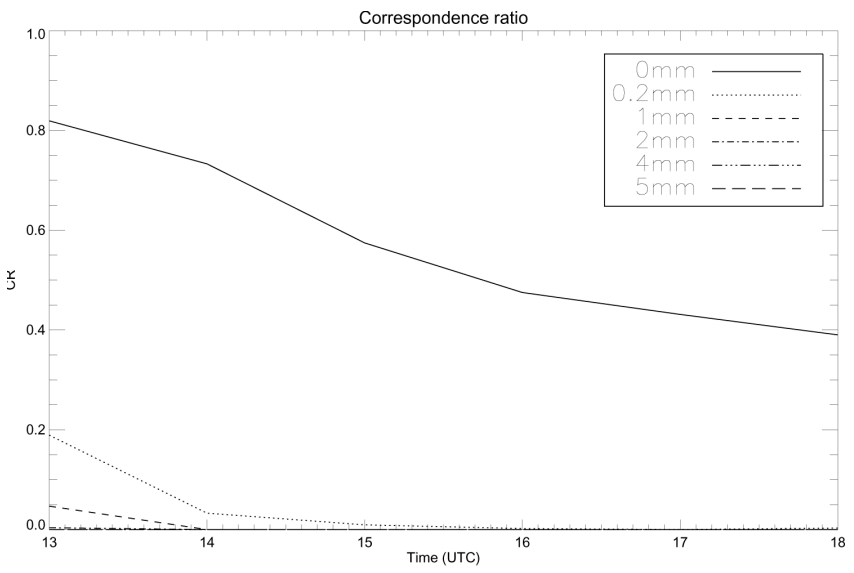

Fig. 11. Correspondence ratio for the control ensemble for hourly rain accumulation.

the radar rain rate. The locations of these localised regions of heavy rain correspond to the two regions of heaviest rain in the ensemble mean at this time (Fig. 9a). By 18:00 UTC the positioning of the rain band in the ensemble mean (Fig. 9d) compares well with the position of the main rain band in the radar (Fig. 5b), although the region of moderately heavy rain off the east coast in the radar is relatively weak in the ensemble mean.

Comparison of the ensemble spread with the innovation magnitude (middle and right columns of Fig. 9) shows that these quantities have quite different spatial distributions at both times shown. In the ensemble spread (Fig. 9b and e) there are more widespread regions of low values of spread, while in the innovation magnitude (Fig. 9c and f) there are more localised regions of higher values. Thus although the domain-averaged quantities (Fig. 10a) appear to be very similar, this is not due to these quantities having the same spatial distribution. The spread-skill plot produced by binning the data by ensemble variance (Fig. 10b) shows generally good agreement between the ensemble forecast error variance and the innovation variance. For larger ensemble forecast error variances the innovation variance is not as large, which could suggest that the ensemble is overspread in areas with higher rain rates. However, this may be at least partly due to the limited ensemble size (Bowler et al., 2008). These results are qualitatively similar to Fig. 16 in Bowler et al. (2008) and Fig. 15 in Migliorini et al. (2011).

The correspondence ratio (CR) was calculated for hourly rainfall accumulation for different accumulation thresholds (Fig. 11). At 13:00 UTC, the CR for the $0 \mathrm{~mm}$ threshold is initially high (0.8), but decreases throughout the forecast, reaching a minimum of around 0.4 . In contrast, for the higher thresholds the CR is initially low (less than 0.2) and quickly decreases to zero or near-zero values. This indicates that after the first $1-2 \mathrm{~h}$ of the forecast there are no, or very few, grid points where all ensemble members agree for these thresholds. These results suggest that, while the ensemble members 

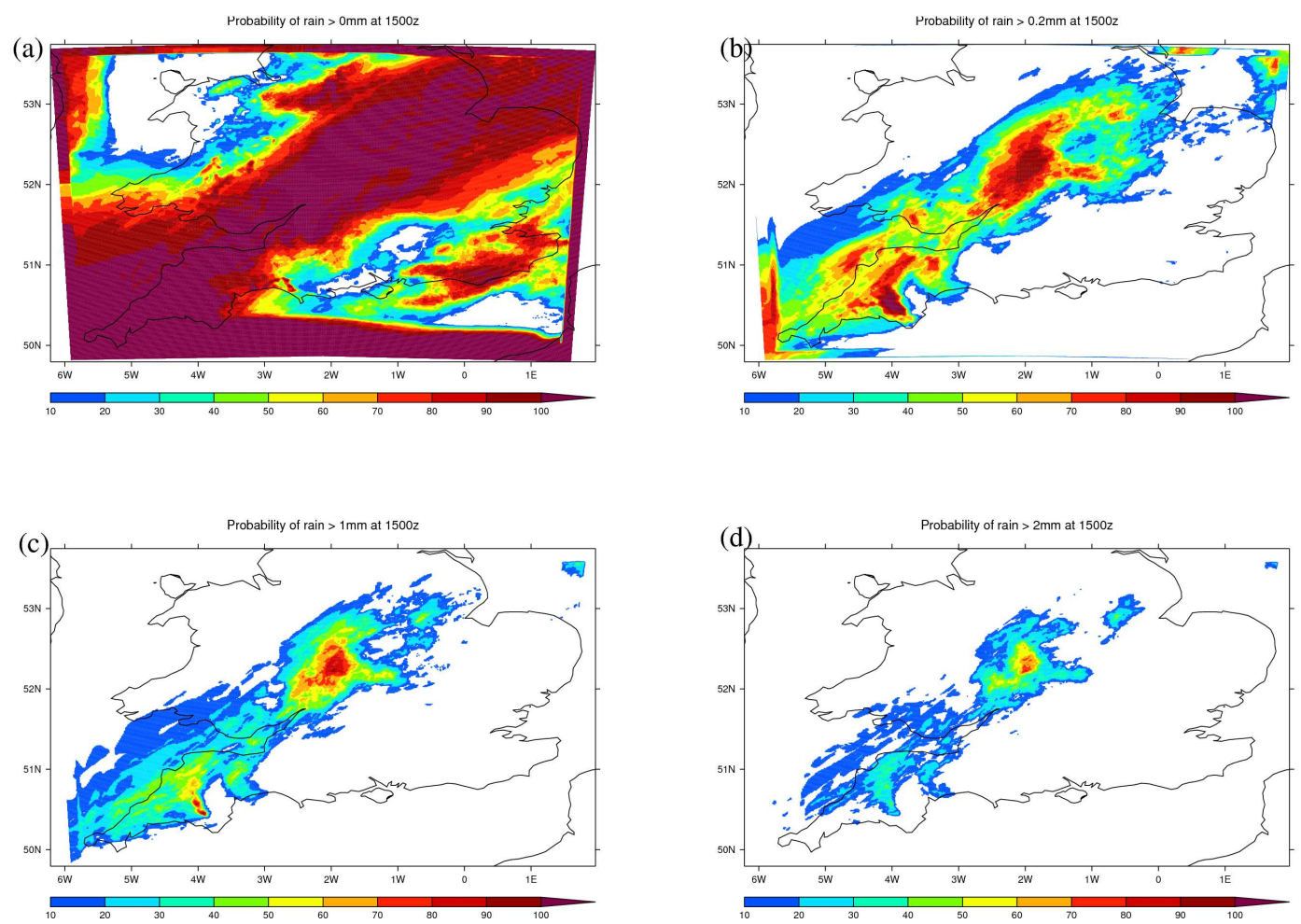

Fig. 12. Probability of rain rate exceeding given thresholds (\%) for the control ensemble at 15:00 UTC. Thresholds are (a) $0 \mathrm{~mm}$, (b) $0.2 \mathrm{~mm}$, (c) $1 \mathrm{~mm}$ and (d) $2 \mathrm{~mm}$.

are in generally good agreement about the location of the rain band, they disagree in the details of the location of heavier rain. This is supported by Fig. 12 which shows the probability of rain exceeding given thresholds. For a $0 \mathrm{~mm}$ threshold (Fig. 12a), there is a broad region across the domain with $100 \%$ probability of rain. In contrast, for higher thresholds (Fig. 12b-d) there are more areas with lower probabilities. For the three thresholds shown in Fig. 12b-d, there is a small region in the centre of the domain with high probabilities. This corresponds to some much smaller regions of rain exceeding $2 \mathrm{~mm}$ in the radar (Fig. 5a). In Fig. 12b and c there is some hint of a slightly separated band extending further north than the main rain band, which may be related to the second band seen in the radar (Fig. 5a), although in Fig. 12b and $\mathrm{c}$ it does not extend as far north.

The Brier score (BS) for hourly rain accumulation (Fig. 13a) shows an improvement in the skill over time for the 0 and $0.2 \mathrm{~mm}$ accumulation thresholds. This improvement over time, particularly after 15:00 UTC, suggests that the information coming through the boundaries at later times improves the forecast. There is also a significant improvement with increasing accumulation threshold, which is largely due to the decrease in the number of points exceeding the larger thresholds (Fig. 13b). This increases the number of points that are correctly forecast as not having rain exceeding the threshold, thus effectively improving the BS.

\section{Evaluation of the effects of applying the RP scheme}

The CTL ensemble represents just one realisation of forecast error. Here we examine the ensembles that have model error variability represented using the RP scheme. The features of each ensemble and the way that each is labelled are described in Sect. 2.5 and in Table 2. Here we show results for one run of each type of ensemble. To confirm that the results presented here are robust and not simply a result of specific combinations of random numbers used in the $\epsilon$ term in Eq. (1), these ensemble runs were repeated using different sets of random numbers. The results from these repeated ensembles were found to be qualitatively similar to those presented here.

One possibility considered is that the different parameters across the ensemble members in all $\mathrm{IC}+\mathrm{BC}+\mathrm{RP}^{*}$ experiments may lead to some members forecasting the multiple rain bands. Once again though, all members in all of these ensembles fail to show this feature. This suggests that it may be the absence of the seed of this feature in the ICs and BCs provided by the driving model - rather than problems associated with model parameter values - that results in this forecast failure, since collectively our experiments have used so many different combinations of model parameters. 
(a)

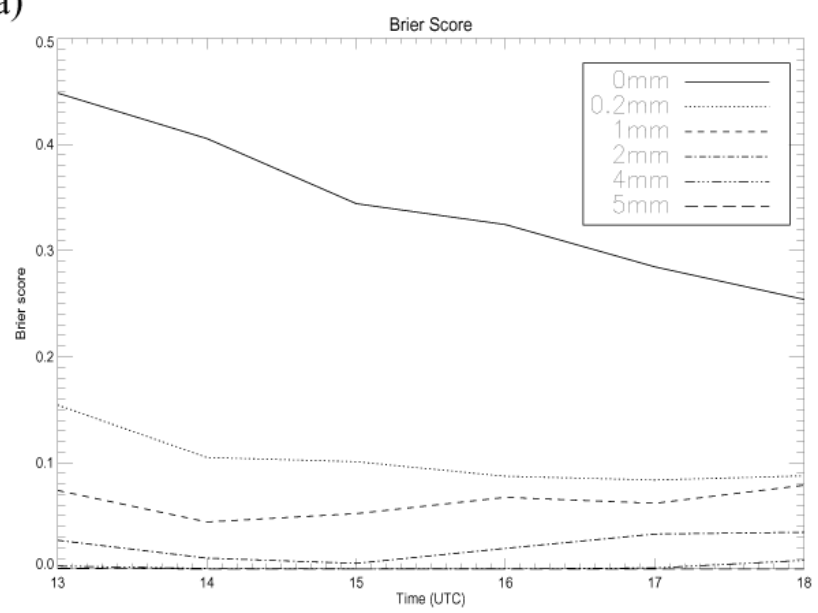

(b)

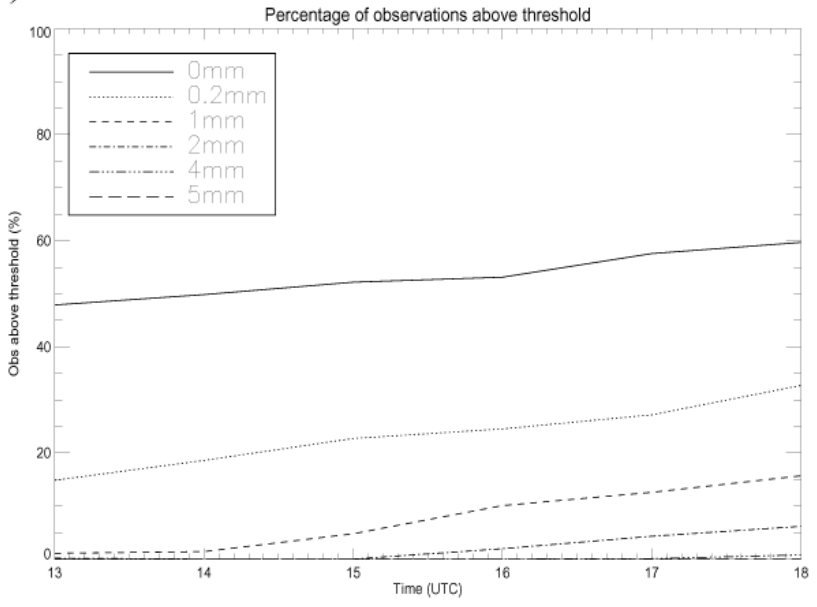

Fig. 13. (a) Brier score for the control ensemble for hourly rain accumulation, calculated using model and radar data resampled to a $13.5 \mathrm{~km}$ grid. (b) shows the number of observation data points exceeding each rain accumulation threshold.

\subsection{The effects of the RP scheme on ensemble spread}

For all the $\mathrm{IC}+\mathrm{BC}+\mathrm{RP}^{*}$ ensembles there is some increase in the ensemble spread compared with the CTL ensemble, for the near-surface fields shown in Fig. 14b-d. For hourly rain accumulation (Fig. 14a) there is a slight decrease in spread in the $\mathrm{IC}+\mathrm{BC}+\mathrm{RP}^{*}$ ensembles compared with the CTL ensemble. Note that the rapid increase in ensemble spread over the first hour of the simulation (until 12:30 UTC) is due to the gradual introduction of the IC perturbations by the IAU scheme over this period, which means that the ensemble members diverge throughout this time. The RP scheme is first applied at the end of the IAU period (at 12:30 UTC), causing a divergence in the spread of the four ensembles at this time. For temperature and $\mathrm{RH}$ (Fig. 14c and d), the three $\mathrm{IC}+\mathrm{BC}+\mathrm{RP}^{*}$ ensembles have consistently larger spread than the CTL ensemble. For these variables there is little difference between the three model error ensembles, although IC $+\mathrm{BC}+\mathrm{RPfix}$ has a slightly larger spread towards the end of the forecast. For $10 \mathrm{~m}$ wind speed (Fig. 14b) there is a slight increase in the spread in the $\mathrm{IC}+\mathrm{BC}+\mathrm{RP}^{*}$ ensembles compared with the CTL ensemble. The spread for the two ensembles with periodic calls to the RP scheme (IC+ BC + RP30 and IC + BC + RP60) has some small jumps at times corresponding to the times that the RP scheme updates the parameters. For hourly rain accumulation (Fig. 14a) the evolution of spread for ensembles IC $+\mathrm{BC}+\mathrm{RP} 30$ and $\mathrm{IC}+\mathrm{BC}+\mathrm{RP} 60$ is less smooth than for ensemble $\mathrm{IC}+\mathrm{BC}+\mathrm{RPfix}$, and while generally lower than the spread for the CTL ensemble, both ensembles have larger spread between around 16:30 and 17:30 UTC than the $\mathrm{IC}+\mathrm{BC}+\mathrm{RPfix}$.

The spread gained by applying the RP scheme compared with the CTL ensemble can be compared with the results of Hacker et al. (2011). Their perturbed parameter ensemble gave an increase in spread for $2 \mathrm{~m}$ temperature of up to $30 \%$ (their Fig. 6a), compared with their control ensemble. This compares well with Fig. 14c, which shows an increase in spread of $30 \%$ in the last hour or so of the forecast for $1.5 \mathrm{~m}$ temperature. Their results also show an increase in spread of around $20 \%$ for $2 \mathrm{~m}$ specific humidity (their Fig. 6c), compared with a $15-20 \%$ increase in the spread for RH (Fig. 14d). In contrast to our results, Hacker et al. (2011) show a negligible change in $10 \mathrm{~m}$ wind speed, and an increase in spread for precipitation accumulation.

Figure $14 \mathrm{a}-\mathrm{d}$ show that model error variability alone introduces much smaller spread than the IC perturbations, but still a much larger amount than the difference between the spread in the $\mathrm{CTL}$ and $\mathrm{IC}+\mathrm{BC}+\mathrm{RP} *$ ensembles. This is consistent with the results of Gebhardt et al. (2011) who found that the effects of their combined physics and LBC perturbations was not the sum of these two perturbations individually. For all four variables shown in Fig. 14a-d the change in spread of the RP* ensembles mirrors the shape of the spread of the $\mathrm{IC}+\mathrm{BC}+\mathrm{RP}^{*}$ ensembles, increasing slightly throughout the forecast. It is interesting to note that the RPfix ensemble still shows an increase in spread throughout the forecast similar to the RP30 and RP60 ensembles, despite the fact that the RP scheme is only applied once. This indicates that the effect of the RP scheme on the spread is due to the members following different model manifolds from the first application, and subsequent changes to the manifolds due to repeated applications of the RP scheme in RP30 and RP60 do not increase the spread further in a substantial way. The similarity of the spreads between all RP* experiments may also be related to the high autocorrelation coefficient, $r$, in Eq. (1) and the nonlinear relationships between the parameter values and the forecasts.

For the $0 \mathrm{~mm}$ threshold (Fig. 15a) the $\mathrm{CR}$ for the $\mathrm{IC}+\mathrm{BC}+\mathrm{RP}^{*}$ ensembles is higher than for the CTL ensemble, indicating a lower spread and a stronger agreement 

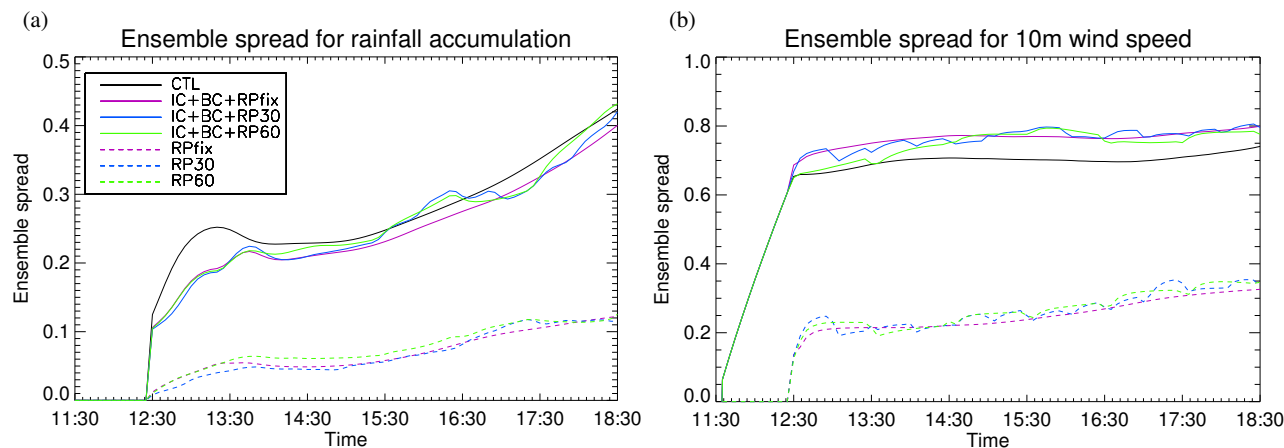

(c)

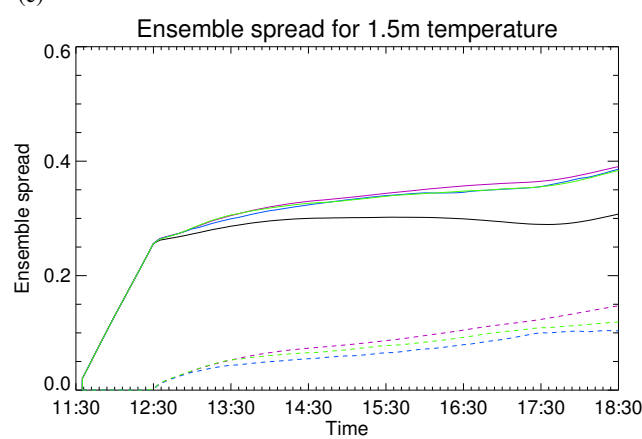

(d)

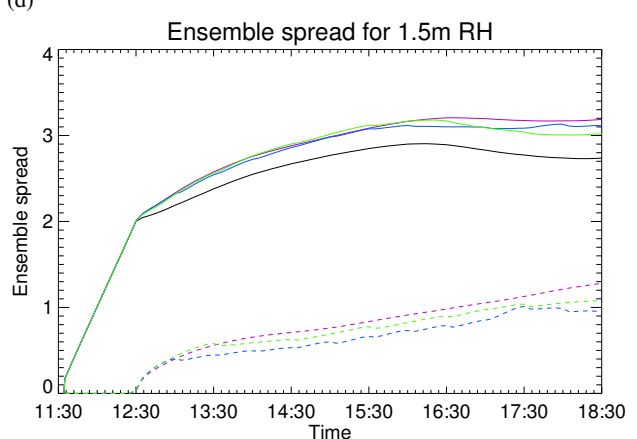

Fig. 14. Evolution of ensemble spread with forecast time for the different ensembles, for (a) hourly rainfall accumulation (mm), (b) $10 \mathrm{~m}$ wind speed $\left(\mathrm{m} \mathrm{s}^{-1}\right)$, (c) $1.5 \mathrm{~m}$ temperature $(\mathrm{K})$ and (d) $1.5 \mathrm{~m} \mathrm{RH}(\%)$.

between members in the location of the rain band. This is in agreement with plots of the probability of rain exceeding $0 \mathrm{~mm}$ for the $\mathrm{IC}+\mathrm{BC}+\mathrm{RP}^{*}$ ensembles (equivalent to Fig. 12a) (not shown) which show broader regions with $100 \%$ probability of rain than for the CTL ensemble. For higher thresholds (Fig. 15b-d) there is no significant change in the $\mathrm{CR}$ for the $\mathrm{IC}+\mathrm{BC}+\mathrm{RP} *$ ensembles compared with the CTL ensemble. The RP* ensembles have higher $\mathrm{CR}$ values than the CTL and $\mathrm{IC}+\mathrm{BC}+\mathrm{RP}^{*}$ ensembles throughout the forecast for all thresholds (Fig. 15a-d). This is consistent with the much lower spread seen in rain accumulation for the RP* ensembles (Fig. 14a). The RP60 ensemble has consistently lower CR values than the other RP* ensembles for all thresholds, indicating more variability between ensemble members in this case. This is in agreement with Fig. 14a which shows a slightly higher spread for the RP60 ensemble than for RP30 and RPfix.

Figure 16a shows that the ensemble mean for the IC + BC + RPfix ensemble has a larger peak and a slightly narrower rain band than the ensemble mean for the CTL ensemble (Fig. 9a). The ensemble spread for the $\mathrm{IC}+\mathrm{BC}+\mathrm{RPfix}$ ensemble extends over a narrower band than the CTL ensemble (Fig. 16b compared with Fig. 9b), which is consistent with the reduction in domain-averaged spread seen in Fig. 14a. The innovation magnitude for the IC + BC + RPfix ensemble (Fig. 16c) is very similar to the innovation magnitude for the CTL ensemble (Fig. 9c), and similarly for the IC $+\mathrm{BC}+\mathrm{RP} 30$ and $\mathrm{IC}+\mathrm{BC}+\mathrm{RP} 60$ ensembles (not shown). Therefore the addition of model error has not improved the relationship between the spatial distributions of ensemble spread and innovation magnitude.

\subsection{The effects of the RP scheme on the forecast skill}

The skill of each ensemble forecast was evaluated using the CRPS, as shown in Fig. 17 (accounting for observation error as described in Sect. 2.4). The observation error variance for each quantity was estimated from spread-skill plots for this case as the value of the skill for zero spread. The values used are specified in the figure caption. Repeating the calculations, but with zero observation error gives qualitatively similar results, which suggests that the results are not sensitive to the exact observation error variance values used.

The CRPS for $1.5 \mathrm{~m}$ temperature and $10 \mathrm{~m}$ winds (Fig. 17c, e and f) shows a general improvement in skill (reduction of CRPS) with forecast lead time, particularly in the $\mathrm{IC}+\mathrm{BC}+\mathrm{RP}^{*}$ ensembles. This is surprising, as skill is normally expected to degrade with lead time. One hypothesis is that this improvement is due to large-scale information (and hence potentially more reliable information) entering the domain via the LBCs. If this were the case, we might expect see reductions in maps of CRPS propagating inwards, especially from the prevailing wind direction (from the south and west boundaries). Also, temporal evolutions of spread-skill relationships at different lead times were calculated to search for evidence of better spread-skill agreement at later lead times. 

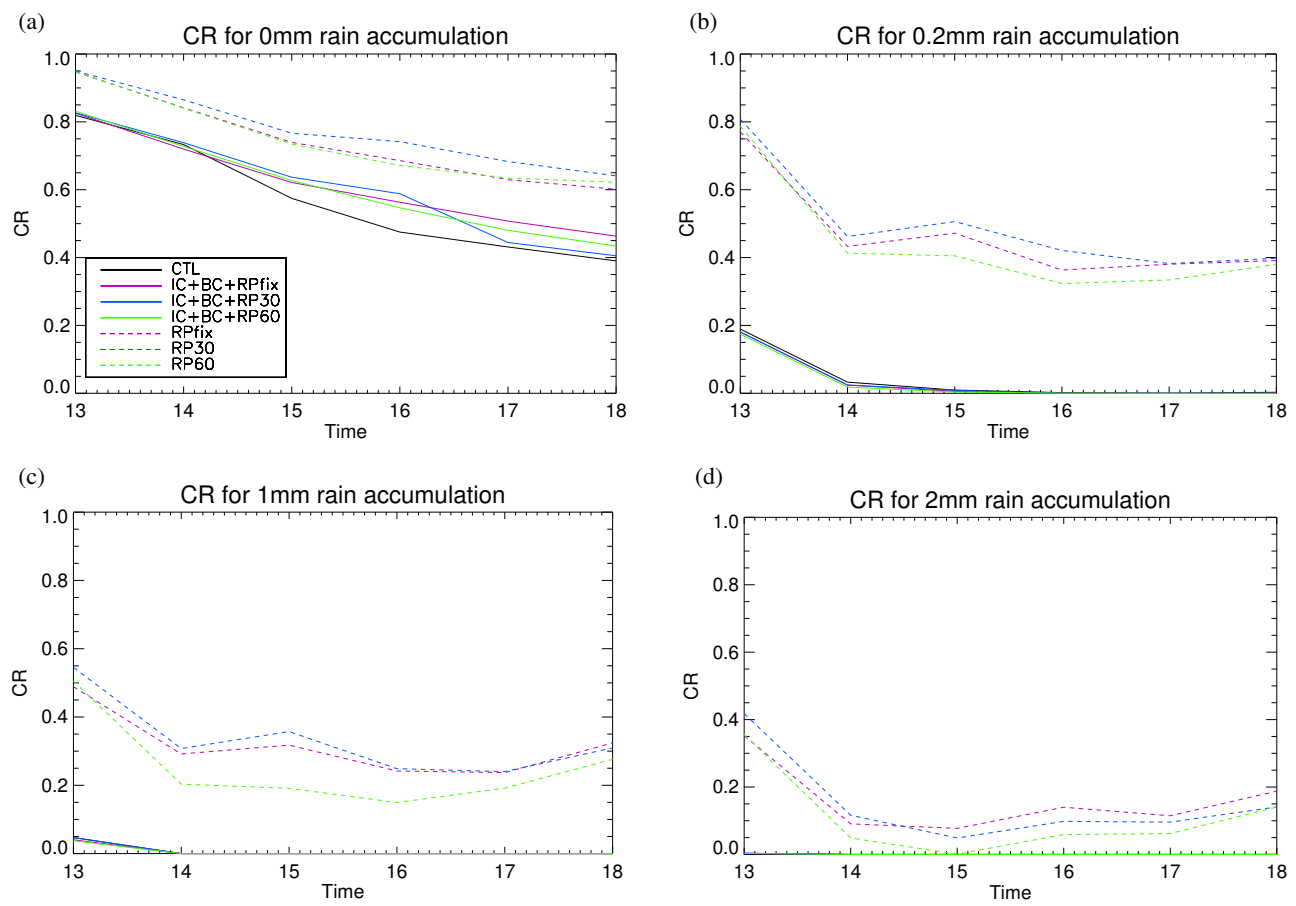

Fig. 15. Correspondence ratio for hourly rain accumulation for all the ensembles. Thresholds are (a) $0 \mathrm{~mm}$, (b) $0.2 \mathrm{~mm},(\mathbf{c}) 1 \mathrm{~mm}$ and (d) $2 \mathrm{~mm}$.

Both investigations, however (results not shown), showed no systematic signals to suggest that the LBC information is responsible for the decrease of CRPS (although this may be due to an insufficient observational sample at any given lead time and so this remains an open question). The improvement in skill with lead time for temperature and winds may be due to the effect of the readjustment of the forecast ensemble to a more balanced set of states after the effect of the inflation on the initial ensemble spread. As discussed in Sect. 2.1, the inflation is a spatially constant factor - determined from temperature and winds power spectra at selected levels - imposed on the ICs as determined by the ETKF. Arguably, a sub-optimal inflation on the initial ensemble spread could cause spurious gravity waves leading to loss of skill in the first hour into the forecast and subsequent improvements in skill would be achieved when the effects of the initial inflation are "forgotten" by the forecast ensemble.

The IC $+\mathrm{BC}+\mathrm{RP}^{*}$ ensembles have consistently better skill than the CTL ensemble, with a significant improvement in skill at and after the $4 \mathrm{~h}$ lead time. For $1.5 \mathrm{~m} \mathrm{RH}$ (Fig. 17d) the skill for all ensembles gets worse over the first two hours, followed by an improvement from the $3 \mathrm{~h}$ lead time. As with $1.5 \mathrm{~m}$ temperature, the $\mathrm{IC}+\mathrm{BC}+\mathrm{RP}^{*}$ ensembles show consistently better skill than the CTL ensemble, with the differences becoming more significant after the $2 \mathrm{~h}$ lead time. For surface $u$ and $v$ wind (Fig. 17e and f) there is large uncertainty in these results, shown by the standard error bars. There is no significant difference in the CRPS between en- sembles, indicating that the RP scheme has little impact on the skill of the wind forecast. For rain rate and hourly rain accumulation (Fig. 17a and b) all ensembles show an improvement in skill between the 1 and $2 \mathrm{~h}$ lead times, followed by a degradation in skill over the remainder of the forecast. For the first $2 \mathrm{~h}$, the $\mathrm{IC}+\mathrm{BC}+\mathrm{RP}^{*}$ ensembles show an improvement in the skill compared with the CTL ensemble, which is particularly significant in the rain accumulation at the $1 \mathrm{~h}$ lead time. After this the $\mathrm{IC}+\mathrm{BC}+\mathrm{RP}^{*}$ ensembles have a significantly worse skill than the CTL ensemble. These results are consistent with results using the PSS (Fig. 18). In the first $2 \mathrm{~h}$ of the forecast the PSS is positive for all thresholds, showing that the $\mathrm{IC}+\mathrm{BC}+\mathrm{RP}^{*}$ ensembles perform slightly better than the CTL ensemble; after this time the PSS is negative, showing that the $\mathrm{IC}+\mathrm{BC}+\mathrm{RP}^{*}$ ensembles are less skillful than the CTL ensemble according to this diagnostic. For the higher thresholds (Fig. 18c and d) the PSS is approximately zero at 15:00 UTC, indicating no change in the skill at this time for these thresholds. All three IC $+\mathrm{BC}+\mathrm{RP} *$ ensembles show similar PSS values throughout much of the forecast, although for the $0 \mathrm{~mm}$ threshold (Fig. 18a) IC + BC + RPfix performs worse than the other ensembles towards the end of the forecast.

The CRPS for the RP* ensembles was also calculated (not shown). These ensembles have consistently worse skill than either the CTL or the IC $+\mathrm{BC}+\mathrm{RP} *$ ensembles. This shows that using the RP scheme to add model error variability alone produces a less skillful ensemble than an ensemble with 

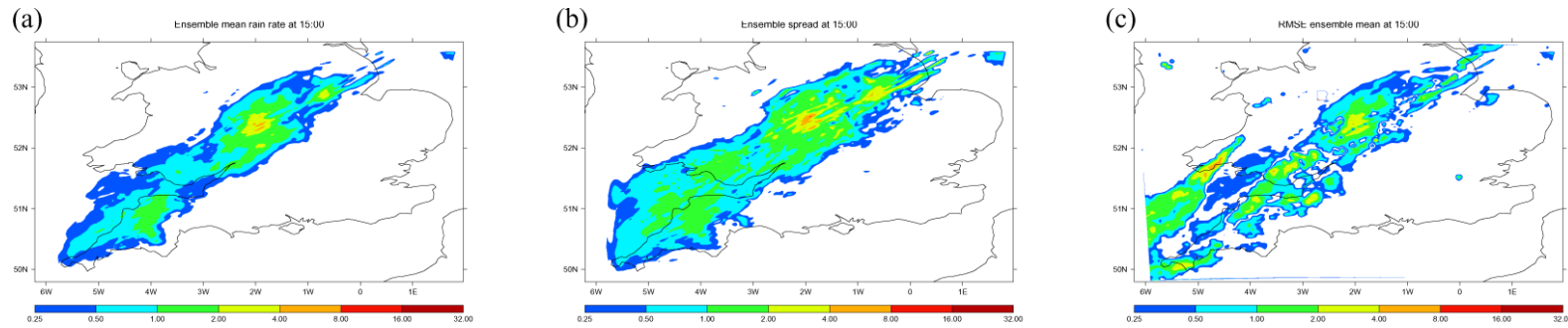

Fig. 16. Rain rate diagnostics for the $\mathrm{IC}+\mathrm{BC}+\mathrm{RPfix}$ ensemble $\left(\mathrm{mm} \mathrm{h}^{-1}\right)$. (a) ensemble mean, (b) ensemble spread and (c) innovation magnitude of the ensemble mean, all at 15:00 UTC 20 September 2011.

perturbed ICs and LBCs. This is likely due to the smaller spread in the RP* ensembles compared with the others.

For all ensembles shown in Fig. 17 (i.e. with and without model error variability) and for the near-surface quantities ( $1.5 \mathrm{~m}$ temperature and $\mathrm{RH}$ and $10 \mathrm{~m}$ wind) the forecasts show a tendency to improve throughout the forecast. This is indicated by a decrease of the CRPS with time. This may be due to the spin-up of the model after the IAU has finished and to large-scale information coming in from the boundaries. All ensembles show an improvement in rainfall skill for the first $2 \mathrm{~h}$ of the forecast, with the $\mathrm{IC}+\mathrm{BC}+\mathrm{RP} *$ ensembles performing better in this period. In contrast, all ensembles show a worsening of skill in rainfall towards the end of the forecast, and the $\mathrm{IC}+\mathrm{BC}+\mathrm{RP} *$ ensembles show worse skill than the CTL ensemble for rain rate and accumulation. This may be due to inconsistencies between fields in the interior of the domain (for which some parameters have been perturbed) and the LBCs (which have not been perturbed). However, the validity of this idea is difficult to demonstrate with just one case study.

\section{Conclusions}

In this paper we have evaluated the effects of using the random parameters (RP) scheme as a method of representing model error in the $1.5 \mathrm{~km}$ EPS for a case on 20 September 2011 in which a frontal rain band crossed through the domain. Although it is difficult to draw conclusions from one case study only, we have shown that the RP scheme in this convective-scale EPS can actually reduce the ensemble variability for some variables.

\subsection{Summary of results}

The standard RP scheme (used operationally in MOGREPS) was used as a basis for the representation of model error uncertainty, and modifications were made to make it suitable for use in the convective-scale $1.5 \mathrm{~km}$ EPS. A set of extra parameters to be perturbed were identified in the large-scale precipitation scheme, in addition to the existing boundary layer and large-scale precipitation parameters (Table 1). Some pairs of related parameters were varied together (using the same ran- dom number), with the intention of controlling each physical process by only one parameter or one pair of parameters. Parameter sensitivity testing on forecasts showed that there was some sensitivity to all the chosen parameters when perturbed individually, and none of the perturbed forecasts gave physically unrealistic results.

Seven ensembles were run with different configurations: the control ensemble had IC and BC perturbations only; three ensembles had IC and BC perturbations and model error represented by the RP scheme, with parameter values updated with different periods; and three ensembles had no IC or BC perturbations but did have model error represented by the RP scheme, with the same three update periods.

The control ensemble was found to represent the main rain band reasonably well, but all members failed to capture the two separate rain bands seen in the radar in the model's domain. The position of the main rain band, however, in this ensemble was positioned in some members at locations occupied by the second rain band in the radar. This was also the case for the ensembles with model error.

The ensembles with IC and BC perturbations and the RP scheme applied were found to have higher domain-averaged ensemble spread for the near-surface temperature, $\mathrm{RH}$ and wind speed than the control ensemble, but a reduced spread for hourly rain accumulation. This decrease in rainfall spread may be due to the fact that the ensembles with the RP scheme applied have a narrower rain band in most members, which accounts for the lower domain averaged spread. The correspondence ratio showed a fast convergence to zero for rain accumulation thresholds greater than zero, indicating that after the first hour or so of the forecast the members disagree on the positions of regions of heavier rain. This is not a surprising result as the regions of heavier rain are found to be more localised than those of lighter rain. The ensembles with the RP scheme applied but no IC and LBC perturbations showed that model error alone introduces less spread than the IC and LBC perturbations (at least in the way that we have configured the RP system), but considerably more spread than the spread gained in the IC $+\mathrm{BC}+\mathrm{RP}^{*}$ ensembles compared with the control. According to the CRPS, applying the RP scheme gave a significant improvement in skill after the first $2-3 \mathrm{~h}$ of the forecast for $1.5 \mathrm{~m}$ temperature and 
(a)

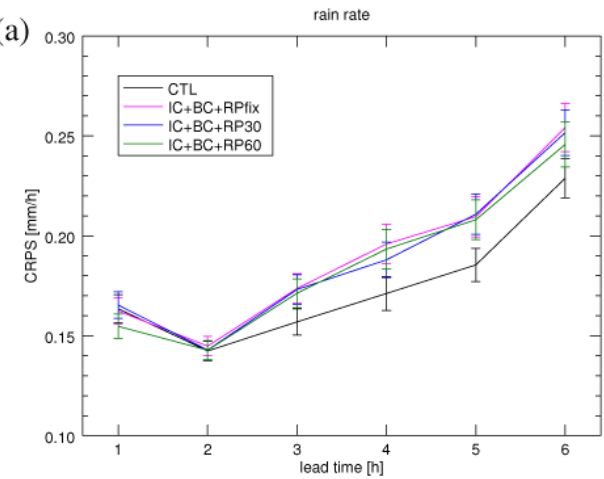

(c)

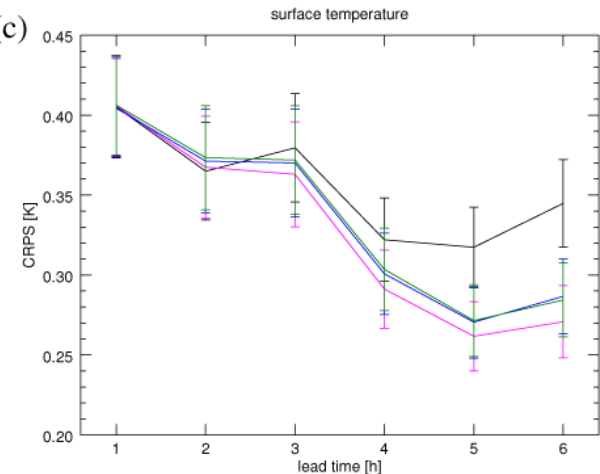

(e)

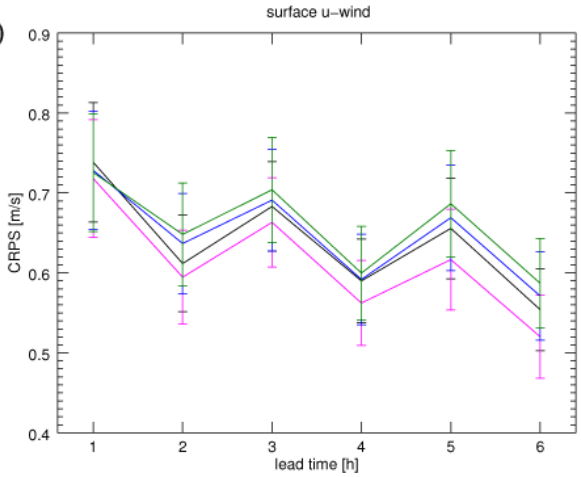

(b)

rain hourly accumulation

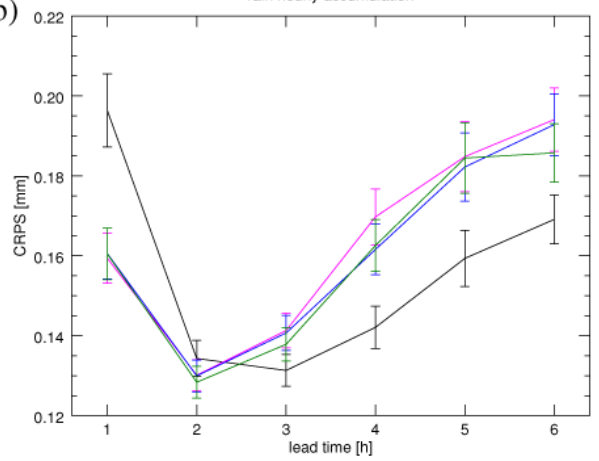

(d)

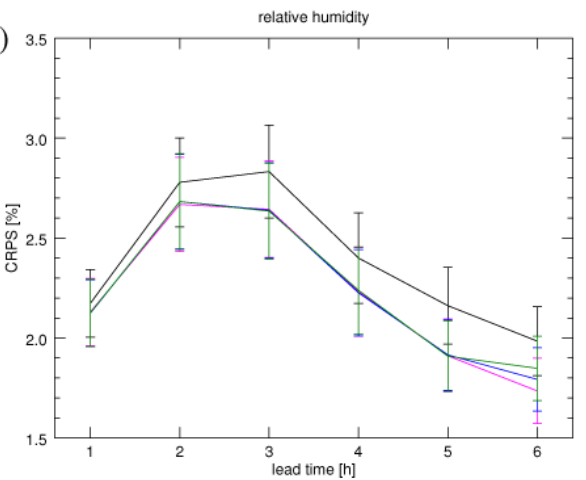

(f)

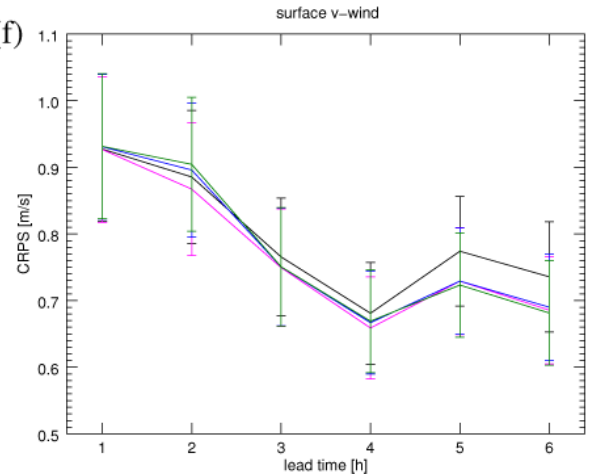

Fig. 17. CRPS for (a) rain rate, (b) hourly rain accumulation, (c) $1.5 \mathrm{~m}$ temperature, (d) $1.5 \mathrm{~m} \mathrm{RH,} \mathrm{(e)} u$ component of $10 \mathrm{~m}$ wind and (f) $v$ component of $10 \mathrm{~m}$ wind. The CRPS shown is the mean CRPS over the domain for each ensemble; error bars show the standard error in the mean. The observation error standard deviations used in the calculation are (a) $\sigma_{\mathrm{o}}=0.47 \mathrm{~mm} \mathrm{~h}^{-1}$, (b) $\sigma_{\mathrm{o}}=0.47 \mathrm{~mm},(\mathbf{c}) \sigma_{\mathrm{o}}=0.69 \mathrm{~K}$, (d) $\sigma_{\mathrm{o}}=4.72 \%$, (e) $\sigma_{\mathrm{o}}=1.54 \mathrm{~m} \mathrm{~s}^{-1}$ and (f) $\sigma_{\mathrm{o}}=1.54 \mathrm{~m} \mathrm{~s}^{-1}$. These values were estimated from spread-skill plots for this case.

$1.5 \mathrm{~m}$ relative humidity, as compared with the control ensemble. For rainfall, there was an improvement in skill at the start of the forecast for the $\mathrm{IC}+\mathrm{BC}+\mathrm{RP}^{*}$ ensembles compared with the control, but a reduced skill in the last few hours. This may be due to inconsistencies between the interior and the boundaries of the domain, which may lead to incorrect rainfall amounts later in the forecast.

The results for both the spread and the skill indicate that perturbing the parameters periodically throughout the forecast has little impact on the forecast spread or skill compared with perturbing the parameters once and holding them fixed for the rest of the forecast. However, perturbing the parameters only once means that less of the parameter space will be spanned.

Overall, for the case discussed here, the effects of applying the RP scheme to an ensemble with IC and LBC perturbations has a positive effect on the spread and skill of nearsurface temperature and relative humidity, but a negative effect on the spread and skill of precipitation. 

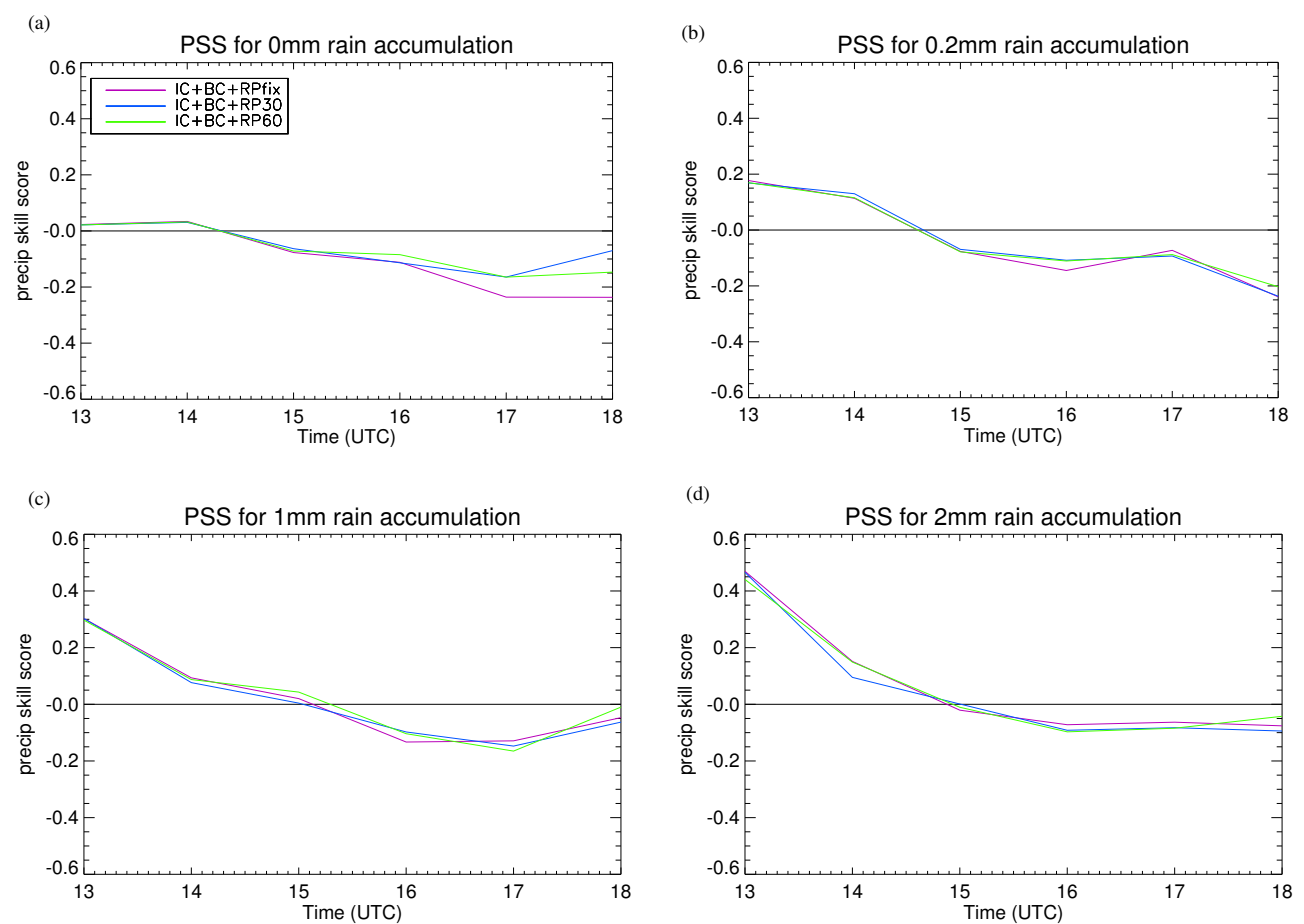

Fig. 18. PSS for hourly rain accumulation for the $\mathrm{IC}+\mathrm{BC}+\mathrm{RP} *$ ensembles with reference to the CTL ensemble for (a) $0 \mathrm{~mm}$, (b) $0.2 \mathrm{~mm}$, (c) $1 \mathrm{~mm}$ and (d) $2 \mathrm{~mm}$ thresholds.

\subsection{Further work}

An obvious limitation of this study is that we have considered only one particular case. This was due to time constraints and upgrades to the modelling system which meant that it was not possible to run further cases with the same model setup. Repeating these experiments with different cases, in particular for different synoptic situations such as convective showers rather than a synoptically forced frontal system would give a broader picture of the usefulness of the RP scheme. We expect that for different synoptic situations there would be differences in the relative sensitivity to each of the parameters. For example, we expect that in a convective case the perturbations made to the large-scale precipitation parameters may have a larger effect than in the case discussed here.

A further limitation is our constraint to using the existing formulation of the RP scheme as a basis for our study. One potential issue with this setup is that each time the parameters are perturbed the model is "kicked" into a different state corresponding to a particular set of parameter values. While the formulation of the autoregression Eq. (1) used in the RP scheme ensures that the parameters remain within a certain range of their previous values, this did still introduce some jumpiness in the wind and rain fields, which is not desirable. An alternative approach would be to update the parameter values more frequently (e.g. every time step, which we might call RPts), but change the autoregression constant $r$ in Eq. (1) to be closer to unity, and/or reduce the range of the shock $\epsilon$.
This would allow a smooth evolution of parameters. By extrapolating the results in this study, we hypothesize that this smoother approach would give a more skillful forecast than the method used here, but may not give any additional increase in ensemble spread. It would also require tuning of $r$ and $\epsilon$, which would be a time-consuming process.

The possible ways in which the RP scheme may be applied raises some interesting issues. At one end of the scale RPfix allows $n$ sets of parameters (where $n$ is the number of ensemble members) to be applied in forecasts that each follow precisely the model equations. At the other end of the scale RPts allows more of parameter space to be explored, but no member will follow the same model equations. Strictly speaking this will lead to loss of continuity (of e.g. mass, water, energy, etc.), but this may actually be a desirable feature given that the model equations are imperfect and the conserved quantities are imperfectly known anyway. Each strategy represents a different philosophical approach to representing model error variability with variable parameters and a comparison would make an interesting piece of future work.

The issues with the degradation of forecast skill for rain rate towards the end of the forecast, which we hypothesize is due to conflicting information in the perturbed domain interior and the information coming in through the domain boundaries from the non-perturbed driving model, suggests that this method may not be ideal for forecasts of more than around a $3 \mathrm{~h}$ lead time. However, the improvement in skill of rainfall forecast in the first $1-2 \mathrm{~h}$ of the forecast show that 
it could be useful for nowcasting and very short forecasts. A potential application of the RP scheme could be to use it within an hourly cycling system as an alternative to the inflation factor as a method of increasing the ensemble spread. It would also be of use on a larger domain, such as that of the new MOGREPS-UK ensemble, which covers the whole of the UK, and therefore the interior of the domain would be less strongly influenced by the LBCs.

Ongoing work aims to investigate the effects of the RP scheme on forecast error covariance statistics and covariance length scales (Migliorini, Bannister, Rudd and Baker, in preparation) and on the inherent balances that are obeyed by the ensemble (Bannister, Migliorini, Baker and Rudd, in preparation). These have applications to informing the appropriate formulation of the background error covariance matrix used in convective-scale data assimilation systems.

Acknowledgements. This work was funded by the Natural Environment Research Council (NERC) under the DIAMET project (Grant ref. NE/I005234/1).The authors wish to thank Ian Boutle and Jonathan Wilkinson for their advice on parameterisation schemes; Emilie Carter, Jean-Francois Caron, Giovanni Leoncini, David Simonin, Simon Wilson and Roger Brugge for technical help; and Sue Ballard, Neill Bowler and Jonathan Flowerdew for helpful discussions on this work. We are also grateful for constructive comments from two anonymous referees and editors of NPG (Roberto Buizza and Olivier Talagrand), which helped to improve the paper. We thank NERC and the Met Office for use of the MetUM and the MONSooN supercomputing facility.

Edited by: R. Buizza

Reviewed by: two anonymous referees

\section{References}

Berner, J., Shutts, G. J., Leutbecher, M., and Palmer, T. N.: A spectral stochastic kinetic energy backscatter scheme and its impact on flow-dependent predictability in the ECMWF Ensemble Prediction System, J. Atmos. Sci., 66, 603-629, 2009.

Berner, J., Ha, S.-Y., Hacker, J. P., Fournier, A., and Snyder, C.: Model uncertainty in a mesoscale ensemble prediction system: Stochastic versus multiphysics representations, Mon. Weather Rev., 139, 1972-1995, 2011.

Bishop, C. H., Etherton, B. J., and Majumdar, S. J.: Adaptive sampling with the ensemble tranform Kalman filter. Part I: Theoretical aspects., Mon. Weather Rev., 129, 420-436, 2001.

Bouttier, F., Vié, B., Nuissier, O., and Raynaud, L.: Impact of Stochastic Physics in a convection-permitting ensemble, Mon. Weather Rev., 140, 3706-3721, 2012.

Bowler, N. E., Arribas, A., Mylne, K. R., Robertson, K. B., and Beare, S. E.: The MOGREPS short-range ensemble prediction system, Q. J. Roy. Meteorol. Soc., 134, 703-722, 2008.

Bright, D. R. and Mullen, S. L.: Short-range ensemble forecasts of precipitation during the Southwest Monsoon, Weather Forecast., 17, 1080-1100, 2002.
Buizza, R., Miller, M., and Palmer, T. N.: Stochastic representation of model uncertainties in the ECMWF Ensemble Prediction System, Q. J. Roy. Meteorol. Soc., 125, 2887-2908, 1999.

Caron, J.-F.: Mismatching perturbations at the lateral boundaries in limited-area ensemble forecasting: A case study, Mon. Weather Rev., 141, 356-374, 2013.

Clark, A. J., Gallus Jr., W. A., Xue, M., and Kong, F.: Growth of spread in convection-allowing and convection-parameterizing ensembles, Weather Forecast., 25, 594-612, 2010.

Clark, A. J., Kain, J. S., Stensrud, D. J., Xue, M., Kong, F., Coniglio, M. C., Thomas, K. W., Wang, Y., Brewster, K., Gao, J., Wang, X., Weiss, S. J., and Du, J.: Probabilistic precipitation forecast skill as a function of ensemble size and spatial scale in a convectionallowing ensemble, Mon. Weather Rev., 139, 1410-1418, 2011.

Davies, T., Cullen, M. J. P., Malcolm, A. J., Mawson, M. H., Staniforth, A., White, A. A., and Wood, N.: A new dynamical core for the Met Office's global and regional modelling of the atmosphere, Q. J. Roy. Meteorol. Soc., 131, 1759-1782, 2005.

Fresnay, S., Hally, A., Garnaud, C., Richard, E., and Lambert, D.: Heavy precipitation events in the Mediterranean: sensitivity to cloud physics parameterisation uncertainties, Nat. Hazards Earth Syst. Sci., 12, 2671-2688, doi:10.5194/nhess-12-26712012, 2012.

Gebhardt, C., Theis, S. E., Paulat, M., and Bouallègue, Z. B.: Uncertainties in COSMO-DE precipitation forecasts introduced by model perturbations and variation of lateral boundaries, Atmos. Res., 100, 168-177, 2011.

Gregory, D. and Rowntree, P. R.: A mass flux convection scheme with representation of cloud ensemble characteristics and stability-dependent closure, Mon. Weather Rev., 118, 14831506, 1990.

Hacker, J. P., Snyder, C., Ha, S.-Y., and Pocernich, M.: Linear and non-linear response to parameter variations in a mesoscale model, Tellus, 63A, 429-444, 2011.

Hersbach, H.: Decomposition of the Continuous ranked probability score for ensemble prediction systems, Weather Forecast., 15, 559-570, 2000.

Lean, H. W., Clark, P. A., Dixon, M., Roberts, N. M., Fitch, A., Forbes, R., and Halliwell, C.: The Met Office global threedimensional variational data assimilation scheme, Q. J. R. Meteorol. Soc., 131, 1759-1782, 2008.

Lin, J. W.-B. and Neelin, J. D.: Influence of a stochastic moist convective parameterization on tropical climate variability, Geophys. Res. Lett., 27, 3691-3694, 2000.

Lock, A. P., Brown, A. R., Bush, M. R., Martin, G. M., and Smith, R. N. B.: A new boundary layer mixing scheme. Part I: scheme description and single-column model tests, Mon. Weather Rev., 128, 3187-3199, 2000.

Migliorini, S., Dixon, M., Bannister, R., and Ballard, S.: Ensemble prediction for nowcasting with a convection-permitting model I: description of the system and the impact of radar-derived surface precipitation rates, Tellus, 63, 468-496, doi:10.1111/j.16000870.2010.00503.x, 2011.

Murphy, J. M., Sexton, D. M. H., Barnett, D. N., Jones, G. S., Webb, M. J., Collins, M., and Stainforth, D. A.: Quantification of modelling uncertainties in a large ensemble of climate change simulations, Nature, 430, 768-772, 2004. 
Shutts, G.: A kinetic energy backscatter algorithm for use in ensemble prediction systems, Q. J. Roy. Meteorol. Soc., 131, 30793102, 2005.

Stainforth, D. A., Aina, T., Christensen, C., Collins, M., Faull, N., Frame, D. J., Kettleborough, J. A., Knight, S., Martin, A., Murphy, J. M., Pianl, C., Sexton, D., Smith, L. A., Spicer, R. A., Thorpe, A. J., and Allen, M. R.: Uncertainty in predictions of the climate response to rising levels of greenhouse gases, Nature, 433, 403-406, 2005.

Stensrud, D. J., Bao, J.-W., and Warner, T. T.: Using initial condition and model physics perturbations in short-range ensemble simulations of mesoscale convective systems, Mon. Weather Rev., 128, 2077-2107, 2000.

Vié, B., Molinié, G., Nuissier, O., Vincendon, B., Ducrocq, V., Bouttier, F., and Richard, E.: Hydro-meteorological evaluation of a convection-permitting ensemble prediction system for Mediterranean heavy precipitating events, Nat. Hazards Earth Syst. Sci., 12, 2631-2645, doi:10.5194/nhess-12-2631-2012, 2012.
Webster, S., Brown, A. R., Cameron, D. R., and Jones, C. P.: Improvements to the representation of orography in the Met Office Unified Model, Q. J. Roy. Meteorol. Soc., 129, 1989-2010, 2003.

Wilks, D. S.: Statistical methods in the atmospheric sciences, Elsevier Academic Press, 2nd Edn., 2006.

Wilson, D. R. and Ballard, S. P.: A microphysically based precipitation scheme for the Meteorological Office Unified Model, Q. J. Roy. Meteorol. Soc., 125, 1607-1636, 1999.

Zadra, A. and Lock, A.: Sensitivity tests with respect to random parameters in the boundary layer scheme, Tech. rep., Met office, 2010. 